Article

\title{
Age-Dependent and Sleep/Seizure-Induced Pathomechanisms of Autosomal Dominant Sleep-Related Hypermotor Epilepsy
}

\author{
Kouji Fukuyama and Motohiro Okada *(D) \\ Department of Neuropsychiatry, Division of Neuroscience, Graduate School of Medicine, Mie University, Tsu, \\ Mie 514-8507, Japan; k-fukuyama@clin.medic.mie-u.ac.jp \\ * Correspondence: okadamot@clin.medic.mie-u.ac.jp; Tel.: +81-59-231-5018
}

Received: 16 October 2020; Accepted: 29 October 2020; Published: 30 October 2020

\begin{abstract}
The loss-of-function S284L-mutant $\alpha 4$ subunit of the nicotinic acetylcholine receptor (nAChR) is considered to contribute to the pathomechanism of autosomal dominant sleep-related hypermotor epilepsy (ADSHE); however, the age-dependent and sleep-related pathomechanisms of ADSHE remain to be clarified. To explore the age-dependent and sleep-induced pathomechanism of ADSHE, the present study determined the glutamatergic transmission abnormalities associated with $\alpha 4 \beta 2$-nAChR and the astroglial hemichannel in the hyperdirect and corticostriatal pathways of ADSHE model transgenic rats (S286L-TG) bearing the rat S286L-mutant Chrna4 gene corresponding to the human S284L-mutant CHRNA4 gene of ADSHE, using multiprobe microdialysis and capillary immunoblotting analyses. This study could not detect glutamatergic transmission in the corticostriatal pathway from the orbitofrontal cortex (OFC) to the striatum. Before ADSHE onset (four weeks of age), functional abnormalities of glutamatergic transmission compared to the wild-type in the cortical hyperdirect pathway, from OFC to the subthalamic nucleus (STN) in S286L-TG, could not be detected. Conversely, after ADSHE onset (eight weeks of age), glutamatergic transmission in the hyperdirect pathway of S286L-TG was enhanced compared to the wild-type. Notably, enhanced glutamatergic transmission of S286L-TG was revealed by hemichannel activation in the OFC. Expression of connexin43 (Cx43) in the OFC of S286L-TG was upregulated after ADSHE onset but was almost equal to the wild-type prior to ADSHE onset. Differences in the expression of phosphorylated protein kinase B (pAkt) before ADSHE onset between the wild-type and S286L-TG were not observed; however, after ADSHE onset, pAkt was upregulated in S286L-TG. Conversely, the expression of phosphorylated extracellular signal-regulated kinase (pErk) was already upregulated before ADSHE onset compared to the wild-type. Both before and after ADSHE onset, subchronic nicotine administration decreased and did not affect the both expression of Cx43 and pErk of respective wild-type and S286L-TG, whereas the pAkt expression of both the wild-type and S286L-TG was increased by nicotine. Cx43 expression in the plasma membrane of the primary cultured astrocytes of the wild-type was increased by elevation of the extracellular $\mathrm{K}^{+}$level (higher than $10 \mathrm{mM}$ ), and the increase in Cx43 expression in the plasma membrane required pErk functions. These observations indicate that a combination of functional abnormalities, GABAergic disinhibition, and upregulated pErk induced by the loss-of-function S286L-mutant $\alpha 4 \beta 2$-nAChR contribute to the age-dependent and sleep-induced pathomechanism of ADSHE via the upregulation/hyperactivation of the Cx43 hemichannels.
\end{abstract}

Keywords: autosomal dominant sleep-related hypermotor epilepsy; L-glutamate; hemichannel; basal ganglia; extracellular signal-regulated kinase; protein kinase B 


\section{Introduction}

In 1994 [1], the first gene mutation associated with idiopathic epilepsy was identified in a pedigree of autosomal-dominant sleep-related hypermotor epilepsy (ADSHE) [2]. Over the past two decades, numerous gene mutations associated with various idiopathic epilepsies have been identified [3]. Until recently, the gene mutations associated with ADSHE were also identified in several genes, such as CHNRA2, CHRNA4, CHRNB2, CRH, KCNT1, and DEPDC5 [2,4,5]. In spite of these efforts, neither the detailed pathomechanisms nor the pathophysiologies of ADSHE have been clarified. To explore pathomechanisms of ADSHE, several genetic ADSHE animal models bearing a missense mutation in the Chrna4 gene, including S280F, insL, and S284L (classical ADSHE-mutations), have been generated. We also studied the pathomechanism/pathophysiology of ADSHE, using transgenic ADSHE rat models, namely S284L-TG, V286L-TG, and S286L-TG [6-11]. Functional analysis, using S286L-TG, which bears an S286L-mutation in the rat Chrna4 gene encoding the $\alpha 4$ subunit of the nicotinic acetylcholine (ACh) receptor (nAChR) corresponding to the S284L-mutation in the human CHRNA4 gene of ADSHE, demonstrated several important functional abnormalities associated with ADSHE and its comorbidity of cognitive deficit [6-8,12].

Recently, we demonstrated two candidate fundamental functional abnormalities associated with the epileptogenesis/ictogenesis of S286L-TG. The first was the loss-of-function S286L-mutant $\alpha 4 \beta 2$-nAChR (enhanced $\alpha 4 \beta 2$-nAChR desensitization and ACh sensitivity), which attenuates GABAergic inhibition in the intrathalamic pathways from the reticular thalamic nucleus (RTN) to motor thalamic nuclei or the mediodorsal thalamic nucleus (MDTN) $[6-8,12]$. The second were the expressions of connexin43 (Cx43) in the frontal cortex (secondary motor cortex) and thalamus (MDTN), which are predominant expression regions of $\alpha 4$-nAChR subunit; they were upregulated in S286L-TG compared with the wild-type littermates [6,12].

The first functional abnormality, impaired GABAergic inhibition in motor thalamic nuclei, plays important roles in the pathomechanisms of typical ADSHE seizures, electroencephalogram (EEG)-sensitive "episodic nocturnal wandering", and "nocturnal paroxysmal arousal" [7,9] via enhanced glutamatergic transmission in the thalamocortical motor pathway (from motor thalamic nuclei to the secondary motor cortex) [6,8]. Impaired GABAergic inhibition in the motor thalamic nuclei also contributes to the pathomechanisms of EEG-insensitive "nocturnal paroxysmal dystonia" via enhanced glutamatergic transmission in the thalamic hypermotor pathway from the motor thalamic nuclei to the subthalamic nucleus (STN), but not via the cortical hyperdirect pathway (from the secondary motor cortex to the STN) [6,8]. Therefore, "nocturnal paroxysmal dystonia" is generated by abnormal intrathalamic GABAergic transmission similar to other EEG-sensitive ADSHE seizure phenotypes, but this may indicate a paroxysmal movement disorder rather than epileptic seizures, as during nocturnal paroxysmal dystonia, the neuronal hyperexcitability is restricted within the thalamus and basal ganglia and does not propagate to the cortex [6-8,12]. These previous studies demonstrated the pathomechanisms of EEG-sensitive "episodic nocturnal wandering" and "nocturnal paroxysmal arousal", as well as EEG-insensitive "nocturnal-paroxysmal-dystonia"; however, these three ADSHE seizures can be comorbid or complicated in the same episodes among individuals. In particular, clinically, "nocturnal paroxysmal dystonia" occurs as an EEG-insensitive paroxysmal movement disorder independently, but it is not rare for this disorder to precede the development of EEG-sensitive "episodic nocturnal wandering" and "nocturnal paroxysmal arousal" [2,13]. Furthermore, even during "episodic nocturnal wandering", it is not rare to be unable to detect ictal discharge when the orbitofrontal cortex (OFC) is the origin of the ictus $[2,13,14]$. ADSHE seizures often feature stereotypical dystonic postures [2,13]. Therefore, the clinical evidence suggests that the epileptic circuits of EEG-insensitive "nocturnal paroxysmal dystonia", EEG-sensitive "episodic nocturnal wandering", and "nocturnal paroxysmal arousal" are likely connected with each other through unknown circuits. In other words, these complicated movement features between EEG-sensitive and EEG-insensitive ADSHE phenotypes suggest that the hyperexcitabilities among the thalamus, cortex, and basal ganglia are likely integrated. According to our hypothesis, to better understand the detailed pathomechanism of ADSHE with 
human S284L-mutations, we should clarify the transmission abnormalities between the cortex and basal ganglia. In the basal ganglia, both the striatum and STN receive the glutamatergic terminal from cortical glutamatergic inputs through the corticostriatal and cortical hyperdirect pathways $[15,16]$. Our previous study did not detect any connectivity between the secondary motor cortex and STN in wild-type and S286L-TG [8]. Cortical connectivity to the STN is sparser compared to that to the striatum, but OFC exhibits higher tract strength for the STN relative to the striatum [17]. Therefore, based on the established neuronal pathway, to explore the functional abnormalities between the frontal cortex and basal ganglia, the present study determined transmission in the corticostriatal (OFC-striatum) and cortical hyperdirect (OFC-STN) pathways, using multi-probe microdialysis systems with an ultra-high-performance liquid chromatograph (UHPLC).

The second functional abnormality that Cx43 expresses in the frontal (secondary motor cortex) and thalamic (MDTN) plasma membrane fraction of S286L-TG was shown to be upregulated rather than the wild-type [6,12]. Furthermore, among wild-type, the activation of $\alpha 4 \beta 2-n A C h R$ suppresses $\mathrm{C} \times 43$ expression in the plasma membrane fraction of the secondary motor cortex; however, $\alpha 4 \beta 2-n A C h R-i n d u c e d$ suppression of Cx43 expression could not be observed in S286L-TG [6]. Connexins are the constituent molecules of hemichannel and Gap-junctions [18]. Cx43 is the most widely and predominantly expressed connexin isoform in astrocytes [18,19]. The hemichannel/connexon is assembled with six connexins, and the connexon on the plasma membrane in two neighboring neurons, along with astrocytes, oligodendrocytes, and microglia, forms a gap-junction [18]. The gap-junction plays important roles in physiological functions, including neuronal excitability, synaptic plasticity, tripartite synaptic transmission, and homeostasis maintenance in the central nervous system $[18,19]$. Astroglial hemichannels also regulate ionic homeostasis, including ionic movement regulation between intracellular and extracellular spaces and the release of several gliotransmitters, including adenosine triphosphate (ATP), nicotinamide adenine dinucleotide, L-glutamate, and prostaglandins, which are involved in autocrine/paracrine signaling $[18,20]$. During the resting stage, the astroglial hemichannels do not contribute to gliotransmitter release due to their low opening probability $[18,20,21]$; however, activated hemichannels induced by depolarization and/or extracellular/intracellular cation mobilization (elevations in extracellular $\mathrm{K}^{+}$and reductions in extracellular $\mathrm{Ca}^{2+}$ ) generate gliotransmitter release $[18,20,21]$. Therefore, hyperactivated astroglial hemichannels are considered to contribute to the generation of epileptic discharges [19,22].

Upregulated $\mathrm{C} \times 43$ in the thalamus and frontal cortex are activated by repetitive/sustained neuronal excitabilities, such as interictal/ictal discharges or physiological benign discharge (sleep spindles) $[6,12,23]$. The enhanced glutamatergic input to the secondary motor pathway plays important roles in the generation of ADSHE focus due to the hyperactivation of the upregulated Cx43 containing hemichannel [6,7]. Therefore, the mechanism by which the loss-of-function S286L-mutant $\alpha 4 \beta 2$-nAChR enhances $C \times 43$ expression in the plasma membrane is another scientific issue that must be clarified. Binding of the agonist to $\alpha 4 \beta 2-\mathrm{nAChR}$ leads to opening with the desensitization of its cation channel, resulting in rapid depolarization on the order of milliseconds. Besides such electrophysiological rapid responses, nAChRs are also recognized to affect long-term intracellular signaling via several pathways [24,25]. The effects of nAChRs on intracellular signaling have been energetically studied as therapeutic targets for Alzheimer's disease and carcinoma [24-27]. It has been established that Cx43 expression in the plasma membrane is regulated by various posttranscriptional processes, including phosphorylation, acetylation, nitrosylation, sumoylation, and ubiquitylation $[18,28]$. Our previous study, using S284L-TG, demonstrated that, before ADSHE onset, chronic administration of furosemide prevents ADSHE onset via the upregulation of the $\mathrm{K}^{+} / 2 \mathrm{Cl}^{-}$cotransporter (KCC) [10]; however, another line of vitro studies also demonstrated that furosemide inhibits mitogen-activated protein kinase (MAPK)/extracellular signal-regulated kinase (Erk) signaling [29]. The Cx43 expression/function is also regulated by several protein kinases, including MAPK/Erk and phosphoinositide 3-kinase (PI3K)/protein kinase B (Akt) pathways [18,30-34]. The activation of $\alpha 7-\mathrm{nAChR}$ enhances the signaling of PI3K/Akt and MAPK/Erk [35,36], whereas the effects of $\alpha 4 \beta 2-n A C h R$ on this signaling remains to 
be clarified [25]. Therefore, to explore the mechanisms of upregulation of Cx43 in S286L-TG, the effects of the subchronic administration of nicotine on the expression of Cx43, phosphorylated Akt (pAkt), and phosphorylated Erk (pErk) were also explored, using a capillary immunoblotting system.

\section{Results}

\subsection{Glutamatergic Transmission Abnormality in the Hyperdirect and Cirticostriatal Pathways Associated with} the Hemichannel before and after ADSHE Onset

It is well-known that, during the resting stage, the hemichannel has low opening probability, but an extracellular cation condition, increased $\mathrm{K}^{+}$, and decreased $\mathrm{Ca}^{2+}$ levels activates hemichannel activity $[6,12,20,21,37]$. According to previous demonstrations, to study the activated hemichannel activity on tripartite synaptic transmission in the OFC, the perfusion medium in the OFC was switched from modified Ringers solution (MRS) to $\mathrm{Ca}^{2+}$-free with $100 \mathrm{mM} \mathrm{K}^{+}$containing modified Ringer's solution (FCHK-MRS), for $20 \mathrm{~min}$ (FCHK-evoked stimulation) [6,8]. To explore the interaction between the effects of hemichannel and $\alpha 4 \beta 2$-nAChR on glutamatergic transmission in the cortical hyperdirect (OFC-STN) and corticostriatal (OFC-striatum) pathways of the wild-type and S286L-TG, before (four weeks of age) and after (eight weeks of age) ADSHE onset, the perfusion medium in the OFC began by using MRS with or without (control) $100 \mu \mathrm{M}$ carbenoxolone (CBX: hemichannel inhibitor), $100 \mu \mathrm{M}$ (E)-N-Methyl-4-(3-pyridinyl)-3-buten-1-amine oxalate (RJR2406: selective $\alpha 4 \beta 2$-nAChR agonist), or $100 \mu \mathrm{M}$ CBX plus $100 \mu \mathrm{M}$ RJR2406. The perfusates in the STN and striatum were maintained with MRS, alone, during the experiment. After the stabilization of the L-glutamate level in the STN or the striatum, the perfusate in the OFC was switched to MRS containing the same agent with $100 \mu \mathrm{M}$ amino-3-(3-hydroxy-5-methyl-isoxazol-4-yl)propanoic acid (AMPA) for $180 \mathrm{~min}$ (first AMPA-evoked stimulation). After the first AMPA-evoked stimulation, the perfusion medium in the OFC was switched to MRS. After the stabilization of the L-glutamate level in the STN or striatum, the perfusion medium in the OFC was switched to FCHK-MRS $\left(\mathrm{Ca}^{2+}\right.$-free with $100 \mathrm{mM} \mathrm{K}^{+}$) for $20 \mathrm{~min}$ (hemichannel activation). After the stabilization of the L-glutamate level in the STN or striatum, the perfusion medium in the OFC was switched to MRS containing the same agent with $100 \mu \mathrm{M}$ AMPA, for $180 \mathrm{~min}$ again (second AMPA-evoked stimulation). The interval between the first and second AMPA-evoked stimulations was around $240 \mathrm{~min}$. The detailed experimental designs are indicated in Section 4.3.

Notably, CBX (non-selective hemichannel and gap-junction inhibitor) rapidly/reversibly inhibits these channels, and is widely used but offer lower permeability through the plasma membrane [38]. Therefore, $\mathrm{CBX}$ is a hemichannel blocker rather than a gap-junction inhibitor; however, $100 \mu \mathrm{M} \mathrm{CBX}$ inhibited the voltage-gated sodium channel [39]. In the present study, CBX was administered, using the reverse dialysis technique [40,41]. The estimated penetration ratio of CBX (molecular weight is 570.8) from the intra to extra dialysis probe was lower than $10 \%$ [42]. Therefore, the concentration of $\mathrm{CBX}$ in the brain tissue around the dialysis probe was lower than $10 \mu \mathrm{M}$.

2.1.1. Glutamatergic Transmission Abnormality in the Hyperdirect and Cirticostriatal Pathways Associated with the Hemichannel after ADSHE Onset (Eight Weeks of Age) (Study_1)

The basal extracellular L-glutamate level in the STN of S286L-TG was larger than that of the wild-type (Figure 1). Perfusion with $100 \mu \mathrm{M}$ AMPA into the OFC increased L-glutamate release in the STN (first AMPA-evoked release) (Figure 1A,D) of S286L-TG, which was larger than that of the wild-type (Figure 1C,F). Hemichannel activation in the OFC induced by perfusion of FCHK-MRS into the OFC for $20 \mathrm{~min}$ did not affect the basal L-glutamate level in the STN of the wild-type (Figure 1A-C) but increased that of S286L-TG (Figure 1D-F), which was reduced by perfusion with $100 \mu \mathrm{M} \mathrm{CBX}$ (hemichannel inhibitor) into the OFC (Figure 1D-F). Hemichannel activation in the OFC enhanced the release of $100 \mu \mathrm{M}$ second AMPA-evoked L-glutamate in the STN of both wild-type and S286L-TG (Figure 1C,F). Inhibition of the hemichannel by perfusion with $100 \mu \mathrm{M} \mathrm{CBX}$ into the OFC inhibited the first AMPA-evoked L-glutamate release in the STN of S286L-TG, without affecting that of the wild-type (Figure 1C,F); however, perfusion with CBX inhibited the second AMPA-evoked L-glutamate release in 
the STN of both wild-type and S286L-TG (Figure 1C,F). Activation of $\alpha 4 \beta 2-n A C h R$ in the OFC induced by perfusion with $100 \mu \mathrm{M}$ RJR2406 into the OFC enhanced both the first and second AMPA-evoked L-glutamate release in the STN of the wild-type, without affecting that of the S286L-TG (Figure 1C,F).
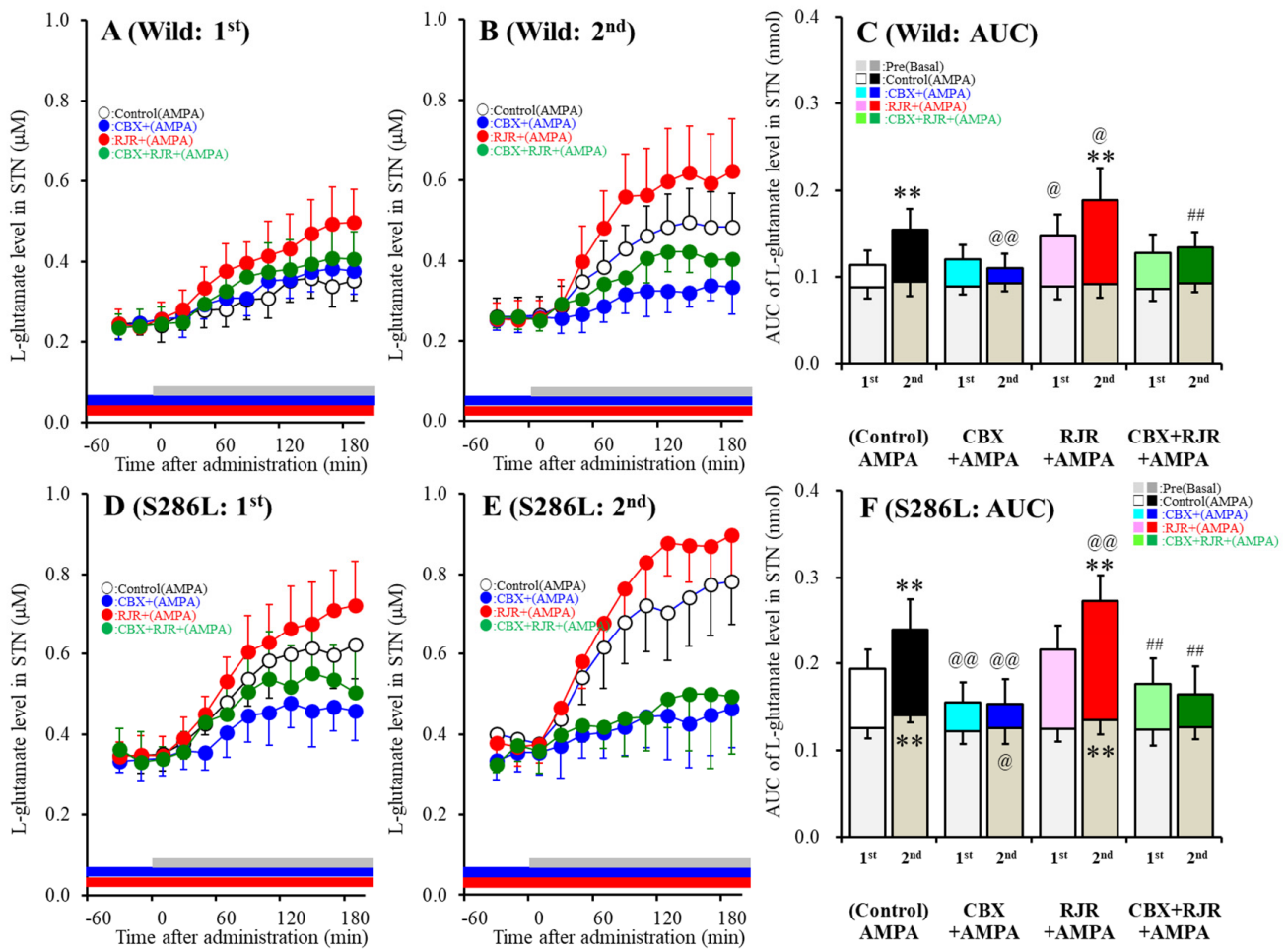

Figure 1. Effects of the local administration of $100 \mu \mathrm{M}$ RJR2403 (selective $\alpha 4 \beta 2$-nAChR agonist) and $100 \mu \mathrm{M}$ carbenoxolone (CBX; hemichannel inhibitor) into the orbitofrontal cortex (OFC) on $100 \mu \mathrm{M}$ amino-3-(3-hydroxy-5-methyl-isoxazol-4-yl)propanoic acid (AMPA)-evoked (perfusion with $100 \mu \mathrm{M}$ AMPA into the OFC) L-glutamate release in the subthalamic nucleus (STN), before (A,D) and after (B,E) hemichannel activation (FCHK-MRS $\left(\mathrm{Ca}^{2+}\right.$-free with $100 \mathrm{mM} \mathrm{K}{ }^{+}$containing modified Ringer's solution) perfusion) of the wild-type (A,B) and S286L-TG (D,E). The perfusion medium in the OFC began with MRS, with or without (control) $100 \mu \mathrm{M}$ CBX (blue bars) or $100 \mu \mathrm{M}$ RJR2403 (red bars). After stabilization of the L-glutamate level in the STN, the perfusion medium was switched to the same MRS containing $100 \mu \mathrm{M}$ AMPA, for $180 \mathrm{~min}$ (first AMPA-evoked stimulation: gray bars). After stabilization of the L-glutamate level in the STN, the perfusion medium was switched from MRS to FCHK-MRS, for $20 \mathrm{~min}$ (hemichannel activation). After stabilization of the L-glutamate level in the STN, the perfusion medium was again switched to the same MRS containing $100 \mu \mathrm{M}$ AMPA, for $180 \mathrm{~min}$ (second AMPA-evoked stimulation: gray bars). Ordinates (A,B,D,E) indicate the mean extracellular L-glutamate level $(\mu \mathrm{M})(n=6)$, and abscissas indicate the time after AMPA-evoked stimulations ( $\mathrm{min})$. (C,F) indicate the area under the curve (AUC) value of the extracellular L-glutamate level (nmol) before (basal extracellular L-glutamate level) and during perfusion with AMPA (from 20 to $180 \mathrm{~min}$ ) of the wild-type (A,B) and S286L-TG (D,E). Notably, the gray columns in $(\mathbf{C}, \mathbf{F})$ indicate the AUC values of the basal extracellular levels of L-glutamate before AMPA-evoked stimulation (basal L-glutamate release) (over -60 to $0 \mathrm{~min}$ in $(\mathbf{A}, \mathbf{B}, \mathbf{D}, \mathbf{E})$ ). ${ }^{*} p<0.05,{ }^{* *} p<0.01$; relative to the first (first AMPA-evoked stimulation), @ $p<0.05$, @ $p<0.01$; relative to the control, \# $p<0.05, \# \# p<0.01$; relative to RJR by MANOVA with Tukey's multiple comparison. The F-values of the L-glutamate level in the STN, according to a multivariate analysis of variance (MANOVA), were $\mathrm{F}_{\text {event }}(1,80)=$ $15.1(p<0.01), \mathrm{F}_{\mathrm{RJR}}(1,80)=17.3(p<0.01), \mathrm{F}_{\mathrm{CBX}}(1,80)=76.8(p<0.01), \mathrm{F}_{\text {genotype }}(1,80)=128.6(p<0.01)$, $\mathrm{F}_{\text {event }}{ }^{*} \operatorname{RJR}(1,80)=0.2(p>0.05), \mathrm{F}_{\text {event }}{ }^{\mathrm{C} B X X}(1,80)=21.3(p<0.01), \mathrm{F}_{\text {event }}{ }^{*}$ genotype $(1,80)=0.1(p>0.05)$, $\mathrm{F}_{\mathrm{RJR} R^{*} \mathrm{CBX}}(1,80)=1.8(p>0.05), \mathrm{F}_{\mathrm{RJR} R^{*} \text { genotype }}(1,80)=0.1(p>0.05), \mathrm{F}_{\mathrm{CBX}}{ }^{*}$ genotype $(1,80)=13.9(p<0.01)$,

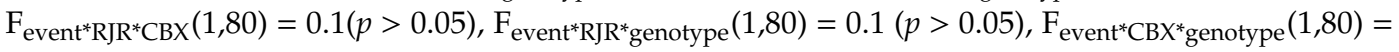
$0.6(p>0.05), \mathrm{F}_{\mathrm{RJR}^{*} \mathrm{CBX} * \text { genotype }}(1,80)=0.1(p>0.05)$, and $\mathrm{F}_{\text {event }}{ }^{*} \mathrm{RJR}^{*} \mathrm{CBX} *$ genotype $(1,80)=0.7(p>0.05)$. 
Contrary to the STN, neither the L-glutamate level in the striatum of S286L-TG nor the wild-type was affected by perfusion with $100 \mu \mathrm{M}$ AMPA and $100 \mu \mathrm{M}$ RJR2403 into the OFC; however, the basal L-glutamate level in the striatum of S286L-TG was larger compared to that of the wild-type (Figure 2). Therefore, the present study could not detect a corticostriatal (OFC-striatum) glutamatergic pathway, whereas glutamatergic neurons are present in the OFC project terminals to the STN (cortical hyperdirect pathway). The cortical hyperdirect glutamatergic transmission receives excitatory $\alpha 4 \beta 2-n A C h R$ in the OFC in the wild-type, whereas the S286L-mutant $\alpha 4 \beta 2$-nAChR in the OFC cannot affect cortical hyperdirect glutamatergic transmission. However, the cortical hyperdirect pathway in S286L-TG was regulated by the activated hemichannel in the OFC compared to that of the wild-type.
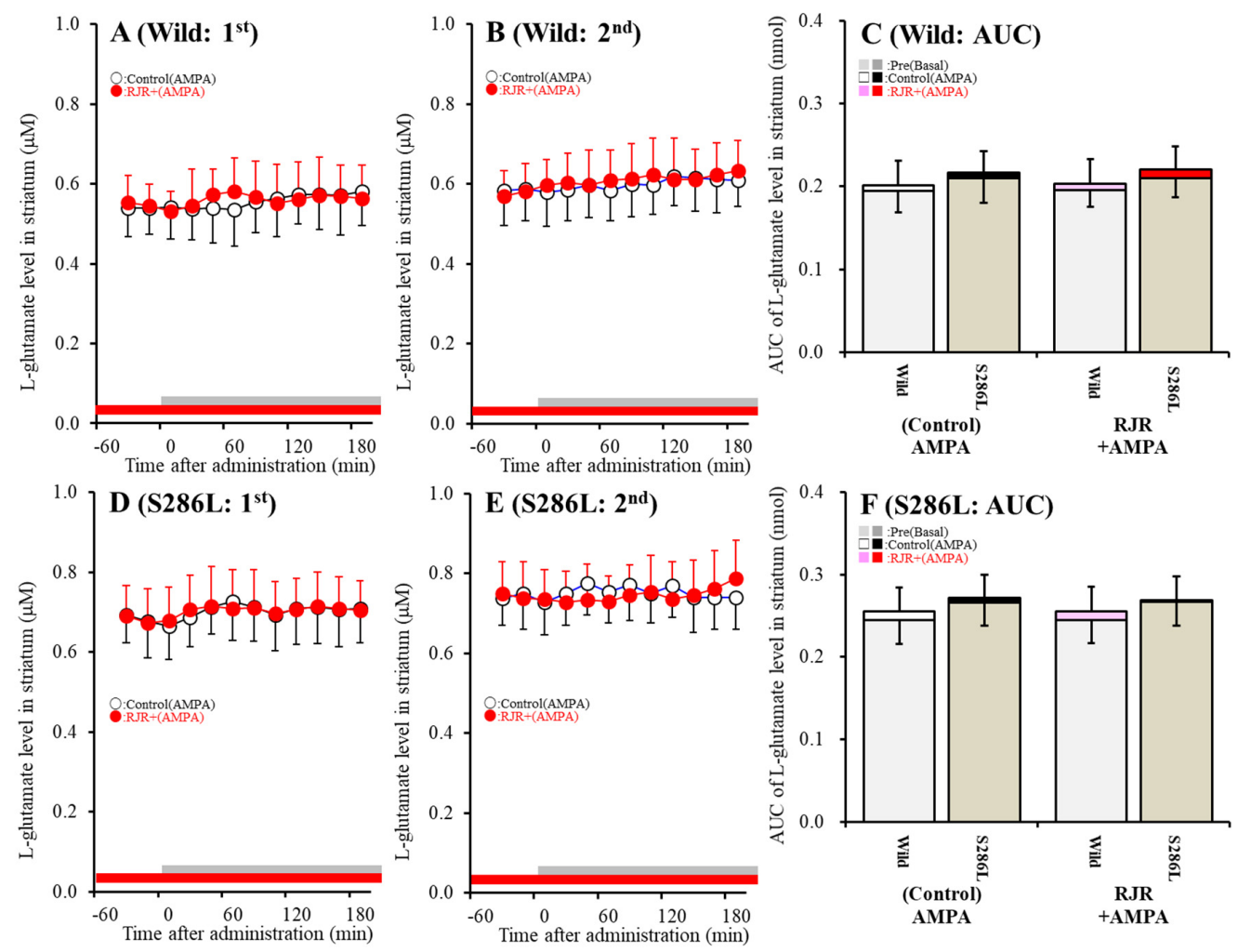

Figure 2. Effects of the local administration of $100 \mu \mathrm{M}$ RJR2403 into the OFC on $100 \mu \mathrm{M}$ AMPA-evoked (perfusion with $100 \mu \mathrm{M}$ AMPA into the OFC) L-glutamate release in the striatum, before (A,D) and after (B,E) hemichannel activation (FCHK-MRS perfusion for $20 \mathrm{~min}$ ) of the wild-type (A,B) and S286L-TG (D,E). The perfusion medium in the OFC began with MRS with or without (control) $100 \mu \mathrm{M}$ RJR2403 (red bars). After stabilization of the L-glutamate level in the striatum, the perfusion medium was switched to the same MRS containing $100 \mu \mathrm{M}$ AMPA for $180 \mathrm{~min}$ (first AMPA-evoked stimulation: gray bars). After stabilization of the L-glutamate level in the striatum, the perfusion medium was switched from MRS to FCHK-MRS for 20 min (hemichannel activation). After stabilization of the L-glutamate level in the striatum, the perfusion medium was again switched to the same MRS containing $100 \mu \mathrm{M}$ AMPA for $180 \mathrm{~min}$ (second AMPA-evoked stimulation: gray bars). Ordinates (A,B,D,E) indicate the mean extracellular L-glutamate level $(\mu \mathrm{M})(n=6)$, and abscissas indicate the time after AMPA-evoked stimulations ( $\mathrm{min})$. (C,F) indicate the AUC of the extracellular L-glutamate level (nmol) before (basal extracellular L-glutamate release) and during perfusion with AMPA (from 20 to $180 \mathrm{~min}$ ) of the wild-type $(\mathbf{A}, \mathbf{B})$ and S286L-TG (D,E), respectively. Notably, the gray columns in $(\mathbf{C}, \mathbf{F})$ indicate the AUC values of the basal extracellular levels of L-glutamate before AMPA-evoked stimulation (basal L-glutamate release) (over -60 to $0 \mathrm{~min}$ in $(\mathbf{A}, \mathbf{B}, \mathbf{D}, \mathbf{E})$ ). The F-values of the L-glutamate level in the striatum by MANOVA were $\mathrm{F}_{\text {event }}(1,40)=3.8(p>0.05), \mathrm{F}_{\mathrm{RJR}}(1,40)=0.1(p>0.05), \mathrm{F}_{\text {genotype }}(1,40)=40.1(p<0.01)$, $\mathrm{F}_{\text {event }{ }^{*} \operatorname{RJR}(1,40)=0.1(p>0.05), \mathrm{F}_{\text {event }}{ }^{*} \text { genotype }}(1,40)=0.1(p>0.05), \mathrm{F}_{\mathrm{RJR}}{ }^{*}$ genotype $(1,40)=0.1(p>0.05)$, $\mathrm{F}_{\mathrm{RJR}}{ }^{*}$ genotype $(1,40)=0.1(p>0.05)$, and $\mathrm{F}_{\text {event*}{ }^{*} \mathrm{RJ}^{*} \text { genotype }}(1,40)=0.1(p>0.05)$. 
2.1.2. Glutamatergic Transmission Abnormality in the Hyperdirect and Cirticostriatal Pathways Associated with the Hemichannel before ADSHE Onset (Four Weeks of Age) (Study_2)

Contrary to after ADSHE onset (eight weeks of age), the basal extracellular L-glutamate level in the STN of S286L-TG prior to ADSHE seizure onset (four weeks of age) was almost equal to that of the wild-type (Figure 3). Both the first and second AMPA-evoked stimulation into the OFC increased L-glutamate release in the STN (Figure 3A,D). The first AMPA-evoked L-glutamate release in the STN of S286L-TG and the wild-type were also almost equal (Figure 3C,F). Perfusion with $100 \mu \mathrm{M} \mathrm{CBX}$ into the OFC did not affect the first AMPA-evoked L-glutamate release but decreased the second AMPA-evoked L-glutamate release in the STN of both S286L-TG and the wild-type (Figure 3C,F). Perfusion with $100 \mu \mathrm{M}$ RJR2406 increased the first AMPA-evoked L-glutamate release in the STN of the wild-type but did not affect that of the S286L-TG; however, the second AMPA-evoked L-glutamate released in the STN of both S286L-TG and the wild-type were enhanced by $100 \mu \mathrm{M}$ RJR2406 (Figure 3C,F). Perfusion with $100 \mu \mathrm{M}$ CBX suppressed the stimulatory effects of $100 \mu \mathrm{M}$ RJR2406 on the second AMPA-evoked L-glutamate release in the STN of both genotypes (Figure 3C,F). Therefore, before ADSHE onset, there were some functional abnormalities in L-glutamate release associated with the hemichannels, whereas the stimulatory effects of $\alpha 4 \beta 2-n A C h R$ on AMPA-evoked L-glutamate release were impaired in S286L-TG.
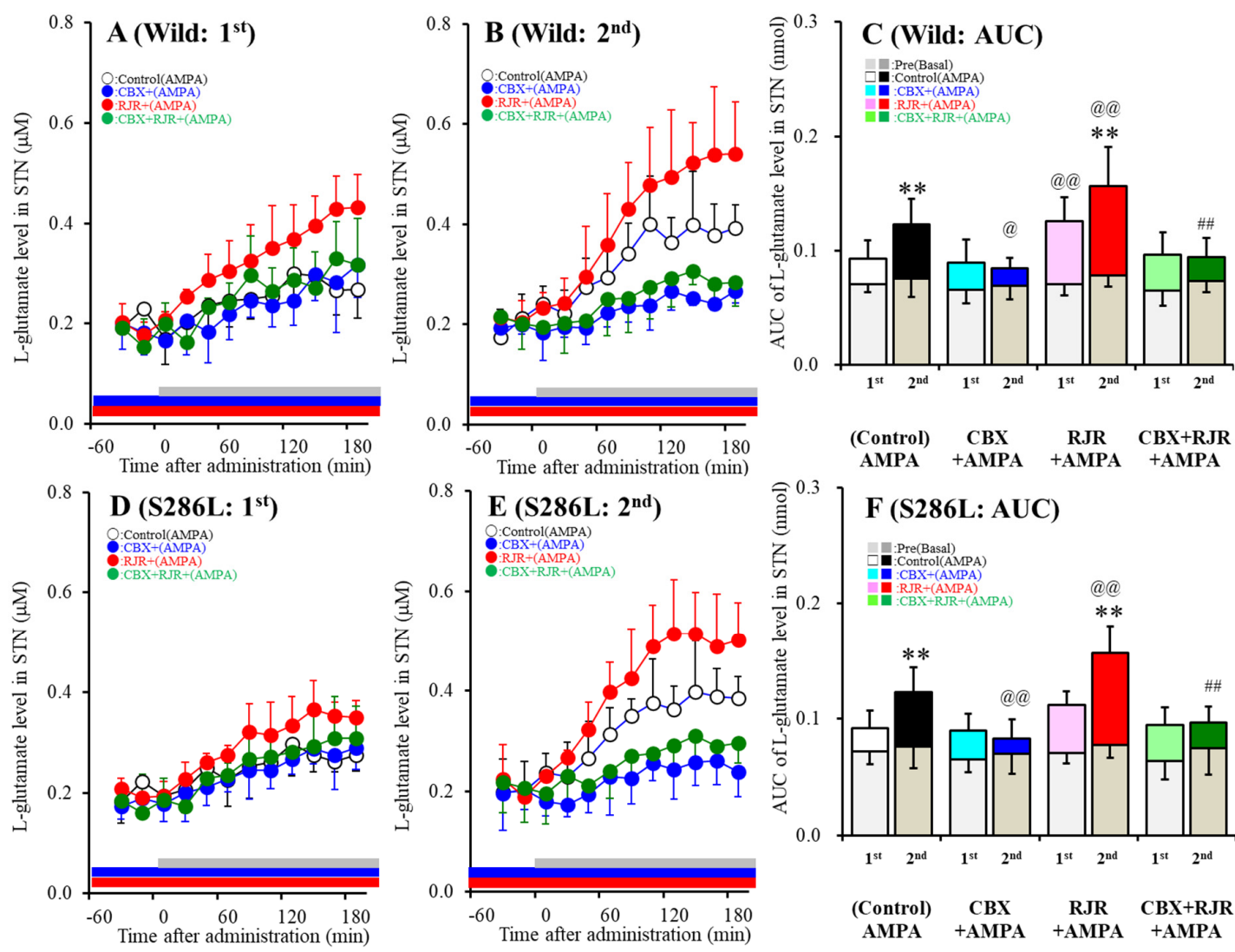

Figure 3. Effects of the local administration of $100 \mu \mathrm{M}$ RJR2403 (selective $\alpha 4 \beta 2$-nAChR agonist) and $100 \mu \mathrm{M} \mathrm{CBX}$ (hemichannel inhibitor) into the OFC on $100 \mu \mathrm{M}$ AMPA-evoked (perfusion with $100 \mu \mathrm{M}$ AMPA into the OFC) L-glutamate release in the STN, before $(\mathbf{A}, \mathbf{D})$ and after $(\mathbf{B}, \mathbf{E})$ hemichannel activation (FCHK-MRS $\left(\mathrm{Ca}^{2+}\right.$-free with $\left.100 \mathrm{mM} \mathrm{K}^{+}\right)$perfusion) of the wild-type $(\mathbf{A}, \mathbf{B})$ and S286L-TG $(\mathbf{D}, \mathbf{E})$. Ordinates $(\mathbf{A}, \mathbf{B}, \mathbf{D}, \mathbf{E})$ indicate the mean extracellular L-glutamate level $(\mu \mathrm{M})(n=6)$, and abscissas indicate the time after AMPA-evoked stimulations (min). (C,F) indicate the AUC value of the extracellular L-glutamate level (nmol) before (basal extracellular L-glutamate level) and during perfusion with AMPA (from 20 to $180 \mathrm{~min}$ ) for the wild-type (A,B) and S286L-TG (D,E), respectively. Especially, gray columns in $(\mathbf{C}, \mathbf{F})$ indicate the AUC values of the basal extracellular levels of L-glutamate before AMPA-evoked stimulation (basal L-glutamate release) (during -60 to $0 \mathrm{~min}$ in $\mathbf{A}, \mathbf{B}, \mathbf{D}, \mathbf{E}$ ). 
${ }^{*} p<0.05,{ }^{* *} p<0.01$; relative to the first (first AMPA-evoked stimulation), @ $p<0.05, @ @ p<0.01$; relative to the control, \# $p<0.05$, \#\# $p<0.01$; relative to RJR by MANOVA with Tukey's multiple comparison. The F-values of the L-glutamate level in the STN, according to a multivariate analysis of variance (MANOVA), were $\mathrm{F}_{\text {event }}(1,80)=15.1(p<0.01), \mathrm{F}_{\mathrm{RJR}}(1,80)=21.9(p<0.01), \mathrm{F}_{\mathrm{CBX}}(1,80)=59.9$ $(p<0.01), \mathrm{F}_{\text {genotype }}(1,80)=0.2(p>0.05), \mathrm{F}_{\text {event }{ }^{*} R J R}(1,80)=0.7(p>0.05), \mathrm{F}_{\text {event }}{ }^{C} \mathrm{CBX}(1,80)=19.3(p<0.01)$, $\mathrm{F}_{\text {event }}^{*}$ genotype $(1,80)=0.3(p>0.05), \mathrm{F}_{\mathrm{RJR} R^{*} \mathrm{CBX}}(1,80)=6.6(p<0.05), \mathrm{F}_{\mathrm{RJR}}{ }^{*}$ genotype $(1,80)=0.1(p>0.05)$,

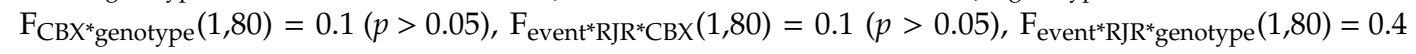
$(p>0.05), \quad \mathrm{F}_{\text {event }}{ }^{*} \mathrm{CBX}{ }^{*}$ genotype $(1,80)=0.1(p>0.05), \mathrm{F}_{\mathrm{RJR}^{*} \mathrm{CBX}{ }^{*} \text { genotype }}(1,80)=0.2(p>0.05)$, and $\mathrm{F}_{\text {event }}{ }^{*} \mathrm{RJ}^{*} \mathrm{CB}{ }^{*}$ genotype $(1,80)=0.1(p>0.05)$.

2.1.3. Tetrodotoxin (TTX) and CBX-Sensitive Basal L-Glutamate Release in the OFC of S286L-TG after Hemichannel Activation (Study_3)

To clarify the mechanisms of enhanced glutamatergic transmission in the cortical hyperdirect pathway induced by hemichannel activation in the OFC of ADSHE onset S286L-TG after FCHK-evoked stimulation in the OFC (after the Study_1), the perfusion medium in the OFC was switched to MRS containing $1 \mu \mathrm{M}$ TTX (voltage-dependent sodium channel inhibitor) [43] or $100 \mu \mathrm{M} \mathrm{CBX} \mathrm{(hemichannel}$ inhibitor). The increased basal L-glutamate release in the OFC after FCHK-evoked stimulation was decreased by CBX but not affected by TTX (Figure 4). Therefore, the increased basal L-glutamate release in the STN of S286L-TG induced by FCHK-evoked stimulation was considered to be of astroglial hemichannel origin but not of neuronal exocytosis origin.

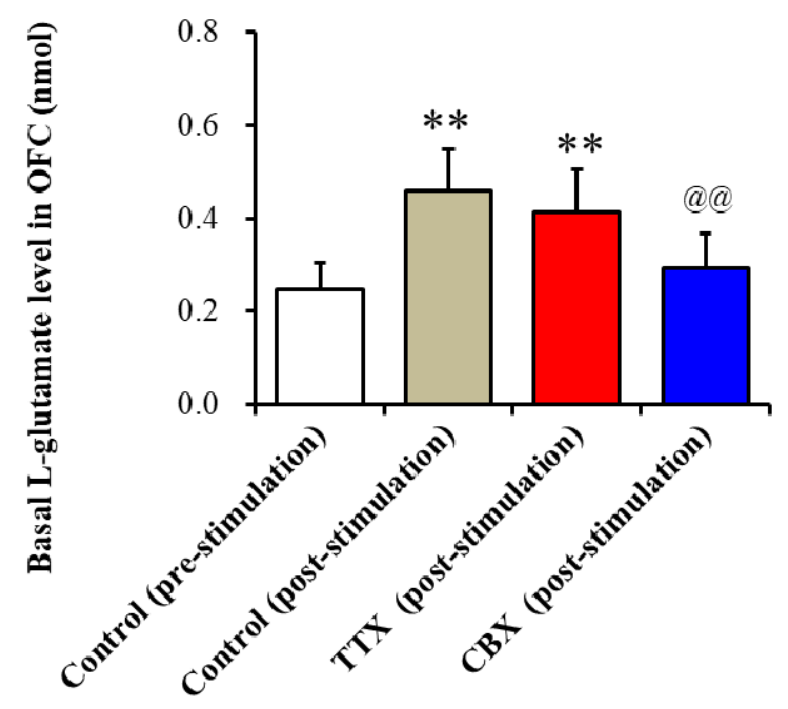

Figure 4. Effects of the local administration of $1 \mu \mathrm{M}$ tetrodotoxin (TTX) and $100 \mu \mathrm{M}$ CBX into the OFC on basal L-glutamate release in the OFC of S286L-TG after FCHK-evoked stimulation. The perfusion medium in the OFC was switched to MRS, with or without (control: post-stimulation: gray column) $1 \mu \mathrm{M}$ TTX (red column) or $100 \mu \mathrm{M} \mathrm{CBX}$ (blue column). The ordinate AUC of the extracellular L-glutamate level (nmol) for 60 min during perfusion of MRS with or without TTX or CBX is shown. ${ }^{* *} p<0.01$, relative to the control (pre-stimulation), and @@ $p<0.01$, relative to the control (post-stimulation) by a one-way analysis of variance (ANOVA) with Tukey's multiple comparison. The F-values of the L-glutamate level in the OFC, according to the one-way ANOVA, are $\mathrm{F}(3,20)=9.6(p<0.01)$.

\subsection{Effects of Subchronic Nicotine Administration on Cx43 Expression in the OFC}

To study the effects of subchronic administration of nicotine on Cx43 expression in the plasma membrane, S286L-TG and the wild-type rats (3 and 11 weeks of ages) were subchronically administrated nicotine (50 mg/kg/day for seven days), using a subcutaneous osmotic pump (2ML_1, Alzet), according 
to a previous study $[6,12,23]$. At four weeks of age (before ADSHE onset), Cx43 expression in the OFC plasma membrane fraction of S286L-TG and the wild-type was almost equal, whereas the subchronic administration of nicotine $(50 \mathrm{mg} / \mathrm{kg} /$ day for seven days) decreased the Cx43 expression of the wild-type but did not affect that of S286L-TG $\left(\mathrm{F}_{\text {genotype }}(1,20)=8.8(p<0.01), \mathrm{F}_{\text {nicotine }}(1,20)=\right.$ $5.7(p<0.05)$, or $\mathrm{F}_{\text {genotype }}$ nicotine $\left.(1,20)=2.7(p>0.05)\right)$ (Figure 5A). Unlike prior to ADSHE onset, at 12 weeks of age (after ADSHE onset), the Cx43 expression of S286L-TG was larger than that of the wild-type (Figure 5B). Subchronic administration of nicotine (50 mg/kg/day for seven days) decreased Cx43 expression of the wild-type but did not affect that of S286L-TG $\left(F_{\text {genotype }}(1,20)=118.0(p<0.01)\right.$, $\mathrm{F}_{\text {nicotine }}(1,20)=17.8(p<0.01)$, and $\left.\mathrm{F}_{\text {genotype*nicotine }}(1,20)=9.6(p<0.01)\right)($ Figure $5 \mathrm{~B})$.
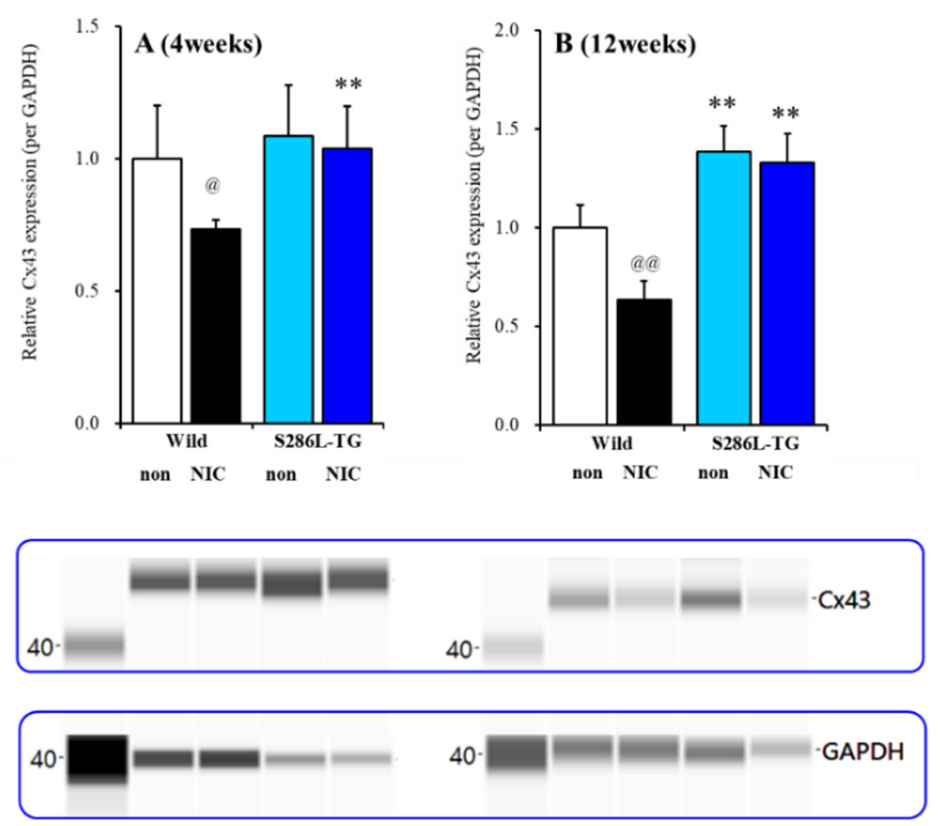

Figure 5. Effects of subchronic nicotine administration on connexin43 (Cx43) expression in the OFC. Effects of systemic subchronic administration of nicotine $(50 \mathrm{mg} / \mathrm{kg} /$ day for seven days) on Cx43 expression in the OFC plasma membrane fraction before four weeks of age (A), and after 12 weeks of age (B), autosomal dominant sleep-related hypermotor epilepsy (ADSHE) onset of the wild-type and S286L-TG and pseudo-gel images from the capillary immunoblotting results, using anti-GAPDH and anti-connexin43 antibodies for blotting of the plasma membrane fractions. Ordinate: mean \pm SD $(n=6)$ of the relative protein level of Cx43. ${ }^{* *} p<0.01$ vs. the wild-type, and @ $p<0.05$, @ $p<0.01$ vs. nicotine-free (non) based on a two-way ANOVA with Tukey's multiple comparison.

\subsection{Effects of Subchronic Nicotine Administration on Akt and Erk Expression in the OFC}

At four weeks of age (before ADSHE onset), phosphorylated Akt (pAkt) expression in the OFC plasma membrane fraction of S286L-TG was almost equal to that of the wild-type; however, at 12 weeks of age (after ADSHE onset), pAkt expression in the OFC plasma membrane fraction of S286L-TG was larger than that of the wild-type (Figure 6A,B). Subchronic administration of nicotine $(50 \mathrm{mg} / \mathrm{kg} /$ day for seven days) increased pAkt expression of the wild-type and S286L-TG before $\left(\mathrm{F}_{\text {genotype }}(1,20)=3.5(p>0.05), \mathrm{F}_{\text {nicotine }}(1,20)=61.3(p<0.01)\right.$, and $\mathrm{F}_{\text {genotype nicotine }}(1,20)$ $=0.1(p>0.05))$ and after $\left(\mathrm{F}_{\text {genotype }}(1,20)=0.1(p>0.05), \mathrm{F}_{\text {nicotine }}(1,20)=103.3(p<0.01)\right.$, and $\mathrm{F}_{\text {genotype }}$ nicotine $\left.(1,20)=18.2(p<0.01)\right)$ the ADSHE onset periods (Figure 6A,B). 

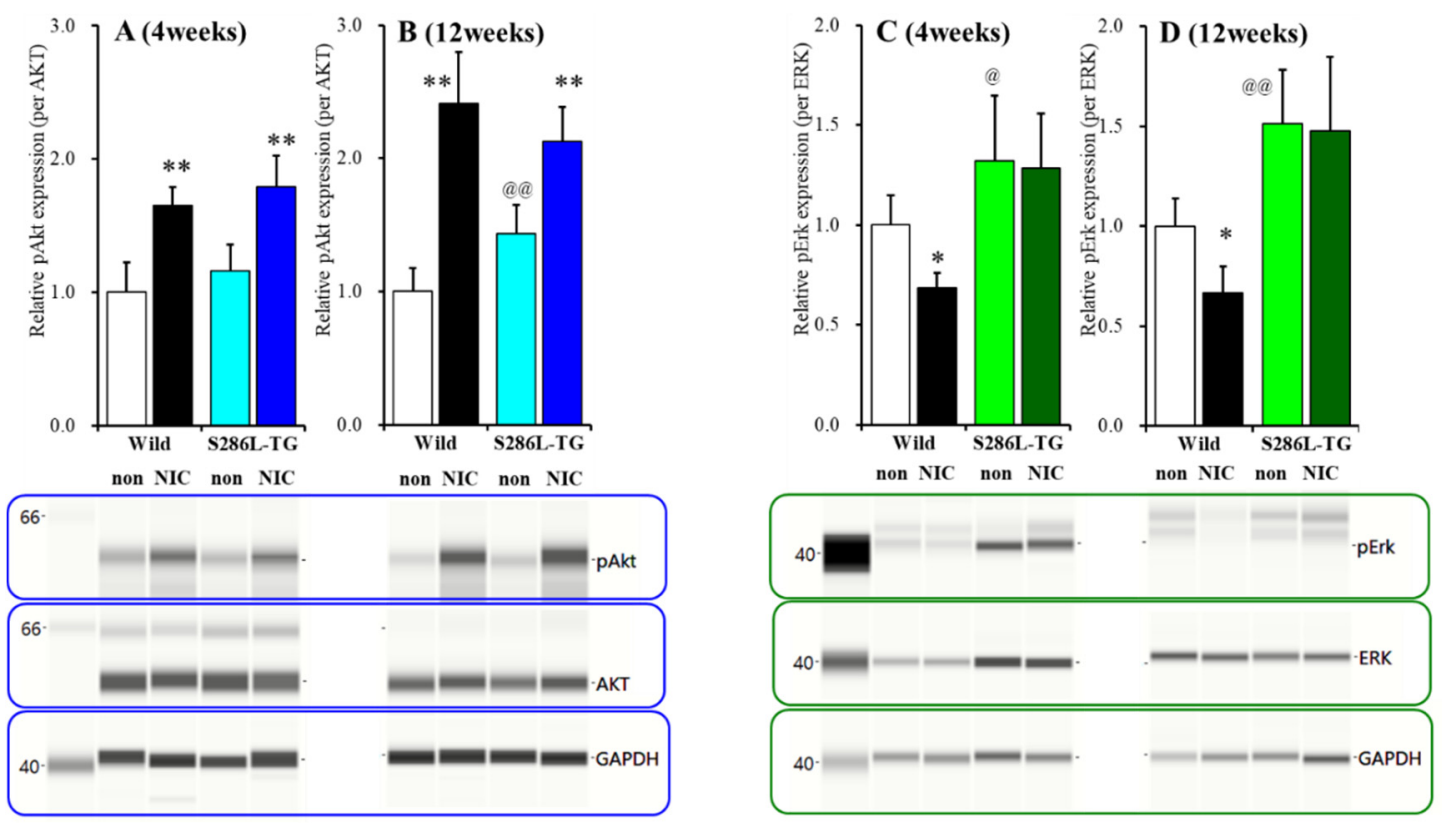

Figure 6. Effects of subchronic nicotine administration on the expression of phosphorylated protein kinase B (pAkt) and phosphorylated extracellular signal-regulated kinase (pErk) in the plasma membrane fraction of OFC. Effects of the systemic subchronic administration of nicotine $(50 \mathrm{mg} / \mathrm{kg} / \mathrm{day}$ for seven days) on pAkt and pErk expression in the OFC plasma membrane fraction before four week of age $(\mathbf{A}, \mathbf{C})$ and after 12 week of age (B,D), ADSHE onset of the wild-type and S286L-TG and pseudo-gel images, using capillary immunoblotting. Ordinate: mean $\pm \operatorname{SD}(n=6)$ of the relative protein level of pErk and pAkt. ${ }^{*} p<0.05,{ }^{* *} p<0.01$ vs. wild-type, and @ $p<0.05$, @ $p<0.01$ vs. nicotine-free (non) by two-way ANOVA with Tukey's multiple comparison.

At four weeks of age, phosphorylated Erk (pErk) expression in the OFC plasma membrane fraction of S286L-TG was already larger than that of the wild-type, whereas subchronic administration of nicotine (50 $\mathrm{mg} / \mathrm{kg} /$ day for seven days) decreased pErk expression of the wild-type but did not affect that of S286L-TG $\left(\mathrm{F}_{\text {genotype }}(1,20)=24.6(p<0.01), \mathrm{F}_{\text {nicotine }}(1,20)=3.7(p>0.05)\right.$, and $\mathrm{F}_{\text {genotype }}$ nicotine $(1,20)=2.3$ $(p>0.05)$ ) (Figure 6C). Like before ADSHE onset, at 12 weeks of age S286L-TG was larger than that of the wild-type (Figure 6D). Subchronic administration of nicotine $(50 \mathrm{mg} / \mathrm{kg} /$ day for seven days) decreased $p$ Erk expression of the wild-type but did not affect that of S286L-TG $\left(F_{\text {genotype }}(1,20)=42.3\right.$ $(p<0.01), \mathrm{F}_{\text {nicotine }}(1,20)=3.3(p>0.05)$, and $\left.\mathrm{F}_{\text {genotype nicotine }}(1,20)=2.1(p>0.05)\right)$ (Figure 6D).

\subsection{Effect of Erk and Extracellular $\mathrm{K}^{+}$Level on Astroglial Cx43 Expression}

After culturing for 28 days (DIV28) to study the effects of the extracellular $\mathrm{K}^{+}$level on Cx43 expression in the plasma membrane, the cultured medium was changed from a Dulbecco's modified Eagle's medium containing 10\% fetal calf serum (fDMEM) to N-fDMEM (control: fDMEM plus $4.6 \mathrm{mM} \mathrm{NaCl}: 5.4 \mathrm{mM}$ $\mathrm{K}^{+}$), MK-fDMEM (fDMEM plus $2.1 \mathrm{mM} \mathrm{KCl}$ and $2.5 \mathrm{mM} \mathrm{NaCl}: 7.5 \mathrm{mM} \mathrm{K}^{+}$), and HK-fDMEM (fDMEM plus $4.6 \mathrm{mM} \mathrm{KCl}: 10.0 \mathrm{mM} \mathrm{K}^{+}$) for $6 \mathrm{~h}$ (around the half-life of $\mathrm{Cx} 43$ [44]). To study the effects of Akt and Erk on $\mathrm{Cx} 43$ expression in the plasma membrane, the cultured medium was changed to HK-fDMEM containing $20 \mu \mathrm{M}$ FR180204 (Erk inhibitor) or $10 \mu \mathrm{M}$ 10-DEBC (Akt inhibitor) for $6 \mathrm{~h}$.

$\mathrm{C} \times 43$ expression in the plasma membrane fraction of the wild-type primary cultured astrocytes increased the extracellular $\mathrm{K}^{+}$level concentration-dependently $(\mathrm{F}(2,15)=27.2(p<0.01))$ (Figure 7A). MK-fDMEM $\left(7.5 \mathrm{mM} \mathrm{K}^{+}\right)$did not affect Cx43 expression, whereas HK-fDMEM $\left(10.0 \mathrm{mM} \mathrm{K}{ }^{+}\right)$increased Cx43 expression in the plasma membrane fraction (Figure 6A). The concentration-dependent expression of the extracellular $\mathrm{K}^{+}$of $\mathrm{Cx} 43$ in the plasma membrane was suppressed by the inhibitor of both Erk (FR180204) and Akt (10-DEBC) $(p<0.01)$ (Figure 7B). 

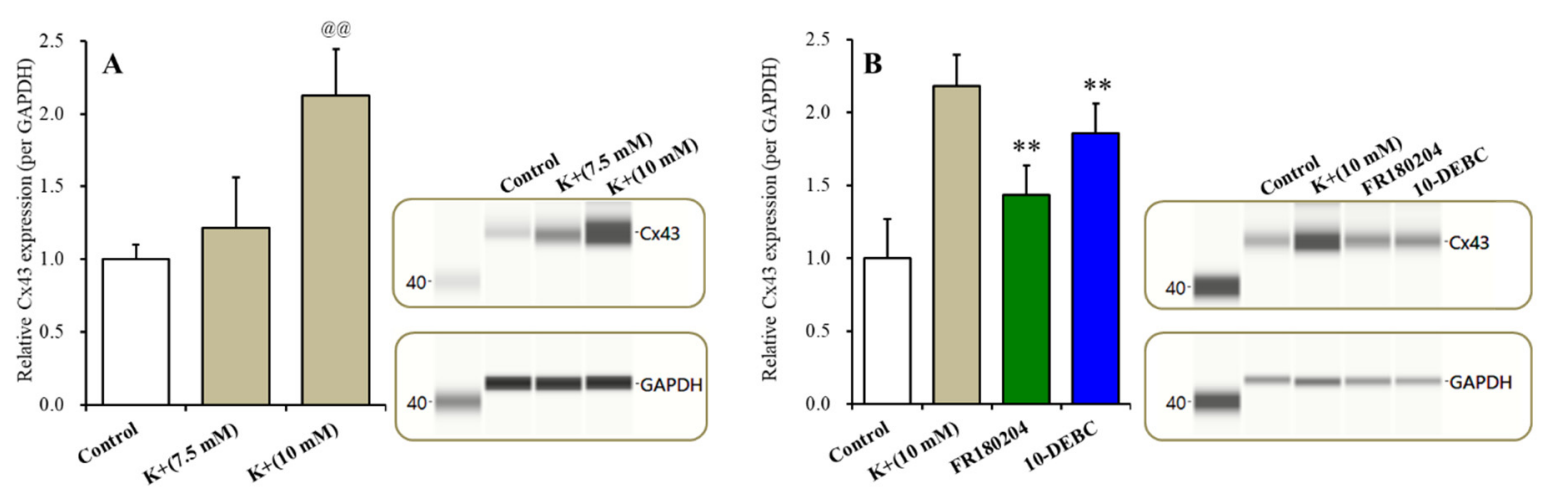

Figure 7. Effects of subacute administration of an increase in the extracellular $\mathrm{K}^{+}$level on Cx43 expression in the plasma membrane fraction of primary cultured astrocytes (A). Effects of the inhibitor of Erk (FR180204) and Akt (10-DEBC) on $\mathrm{K}^{+}$-dependent Cx43 expression in the plasma membrane (B). Ordinate: mean $\pm \mathrm{SD}(n=6)$ of the relative protein level of $\mathrm{Cx} 43$. Concentration-dependent effects of extracellular $\mathrm{K}^{+}$on $\mathrm{Cx} 43$ expression in the plasma membrane fraction of the primary cultured astrocytes were analyzed by a one-way ANOVA (@@ $p<0.01$ vs. control). The effects of $20 \mu \mathrm{M}$ FR180204 and $10 \mu \mathrm{M} 10$-DEBC on Cx43 expression in the plasma membrane fraction were analyzed by a Student's $t$-test $\left(* * p<0.01\right.$ vs. $\left.10 \mathrm{mM} \mathrm{K}^{+}\right)$.

\section{Discussion}

\subsection{Mechanisms of Upregulation of Cx43 of S286-TG.}

We have already demonstrated that a combination of attenuated intrathalamic GABAergic transmission and upregulated/hyperactivated Cx43 in the secondary motor cortex and thalamus of S286L-TG plays important roles in the pathomechanisms of ADSHE [6-8,12]. The propagation of epileptic discharge in the thalamocortical cognitive (MDTN-OFC) pathway was highly dependent upon $\mathrm{Cx} 43$ upregulation/hyperactivation in the thalamus compared to that in the thalamocortical motor pathway (from the motor thalamic nuclei to the secondary motor cortex) $[8,12]$. The present study also detected the upregulation of Cx43 in the OFC plasma membrane of S286L-TG.

Activation of $\alpha 4 \beta 2-n A C h R$ suppressed Cx43 expression [6,45]; however, the loss-of-function S286L-mutant $\alpha 4 \beta 2$-nAChR could not affect Cx43 expression in S286L-TG [6,12]. Trafficking of Cx43 to the plasma membrane was regulated by various posttranscriptional processes (phosphorylation, acetylation, nitrosylation, sumoylation, and ubiquitylation) [18,28], including PI3K/Akt and MAPK/Erk signaling [18,34,46]. Indeed, in the present study, the inhibitors of MAPK/Erk (FR180204) and PI3K/Akt (10-DEBC) suppressed Cx43 expression in the plasma membrane fraction of the primary cultured astrocytes [33,46]. Homomeric $\alpha 7-\mathrm{nAChR}$ stimulated proliferation via the activation of the signaling of PI3K/Akt and MAPK/Erk $[24,25,35,36]$. Contrary to $\alpha 7-n A C h R, \alpha 4 \beta 2-n A C h R$ inhibits proliferation, but its effects on PI3K/Akt and MAPK/Erk signaling remain to be clarified [24-26]. Nicotine inhibits the mRNA of MAPK and PI3K, but the inhibitory effects of nicotine administration were attenuated age-dependently [27]. These age-dependent effects of nicotine on intracellular signaling associated with the PI3K/Akt and MAPK/Erk pathways suggest possible involvement in the age-dependent pathomechanisms of ADSHE. Based on these previous findings, we hypothesized that $\mathrm{Cx} 43$ upregulation in $\alpha 4 \beta 2-\mathrm{nAChR}$-predominant expression regions, the thalamus and frontal cortex of S286L-TG, is likely generated by abnormalities in signaling associated with PI3K/Akt and/or MAPK/Erk induced by the loss-of-function S286L-mutant $\alpha 4 \beta 2-n A C h R$.

According to our expectations, before ADSHE onset (four weeks of age), Cx43 expression in the plasma membrane of S286L-TG OFC was almost equal to that of the wild-type; however, after ADSHE onset (12 weeks of age), Cx43 expression in the plasma membrane of S286L-TG was upregulated compared to that of the wild-type. Subchronic nicotine administration reduced Cx43 expression of the wild-type at both 4 and 12 weeks of age, whereas nicotine did not affect the Cx43 expression of S286L-TG before and 
after ADSHE onset. These results suggest that the loss-of-function S286L-mutant $\alpha 4 \beta 2$-nAChR plays important roles in the pathomechanisms of ADSHE via the upregulation of astroglial Cx43.

Contrary to Cx43 expression, the pErk of S286L-TG was already upregulated before ADSHE onset compared to that of the wild-type. Subchronic nicotine administration reduced the pErk of the wild-type at both 4 and 12 weeks of age, whereas nicotine did not affect the pErk of S286L-TG before and after ADSHE onset. Furthermore, our previous study already demonstrated that furosemide, which prevents the ADSHE onset of S284L-TG [10], inhibits MAPK/Erk signaling [29]. Thus, considering our previous demonstration, the upregulation of pErk preceding Cx43 upregulation in the OFC of S286L-TG suggests the possible pathomechanisms of ADSHE though which the upregulation of MAPK/Erk signaling induced by the loss-of-function S286L-mutant $\alpha 4 \beta 2$-nAChR plays key roles in the development of the epileptogenesis of ADSHE.

Differences between pAkt expression of the wild-type and S286L-TG were not observed before the ADSHE onset periods; however, after ADSHE onset, the pAkt of S286L-TG was upregulated compared to that of the wild-type. Conversely, sub-chronic nicotine administration increased the pAkt of both wild-type and S286L-TG before and after the ADSHE onset periods. This nicotine-induced upregulation of pAkt is generated by the nicotine-induced activation of $\alpha 7-n A C h R$, which is consistent with a previous demonstration $[24,25,35,36]$. Whether the upregulation of pAkt after ADSHE onset is pathomechanism or a result of ADSHE seizure is a fundamental neuroscientific issue. It is well-known that enhanced IP3K/Akt signaling plays a key role in the development of the epileptogenesis and ictogenesis of various epileptic syndromes via suppression of the tuberous sclerosis complex (TSC) and activation of mammalian target of rapamycin (mTOR) signaling [47]. In other line aspects, epileptic seizure also upregulates PI3K/Akt/mTOR signaling [48]. Based on these previous findings, the present study cannot assert that pAkt is not involved in the pathomechanism of ADSHE, but the upregulation of pErk may play a more important role in the development of the epileptogenesis of ADSHE than in pAkt signaling.

\subsection{Impact of the Upregulation/Activation of Cx43 in ADSHE.}

During the resting stage, the astroglial hemichannel exhibits a low opening probability $[6,12,20,21]$ but is activated by the depolarization of membrane potential and specific fluctuations in the extracellular and intracellular cation levels $[6,12,21]$. A functional analysis study using S284L-TG demonstrated that impaired GABAergic inhibition and interictal discharge onset are observable at four and six weeks of age (both before ADSHE onset), respectively $[10,11]$. Electrophysiological studies of Xenopus oocytes, using voltage-clamps, showed that the S284L-mutant $\alpha 4 \beta 2$-nAChR enhances ACh-sensitivity and desensitization [49]. Therefore, the enhanced irritability (repetitive/persistent excitability) induced by impaired GABAergic inhibition via the loss-of-function S286L-mutant $\alpha 4 \beta 2$-nAChR can activate hemichannel functions [6,12]. In our previous microdialysis studies, $25 \mathrm{mM} \mathrm{K}^{+}$-evoked stimulation that could increase neurotransmitter release [50], but gliotransmitter release required greater than $100 \mathrm{mM}$ extracellular $\mathrm{K}^{+}$levels [12,37]. However, elevation of the extracellular $\mathrm{K}^{+}$level around 10-12 $\mathrm{mM}$ plays an important role in the generation of hypersynchronous neuronal excitability, and epileptic discharge also increases the extracellular $\mathrm{K}^{+}$level to over $10 \mathrm{mM}$ [51]. Based on these previous findings, to explore the repetitive/persistent elevation of the extracellular $\mathrm{K}^{+}$level (over $10 \mathrm{mM}$ ) on astroglial hemichannel activity, the effects of subacute (for $6 \mathrm{~h}$, longer than the half-life of Cx43 [44]) exposure to the $10 \mathrm{mM}$ extracellular $\mathrm{K}^{+}$level on $\mathrm{Cx} 43$ expression in the plasma membrane of the wild-type primary cultured astrocytes were studied. According to our expectations, astroglial $\mathrm{Cx} 43$ expression was increased by elevation of the extracellular $\mathrm{K}^{+}$level $\left(7.5 \mathrm{mM} \mathrm{K}^{+}\right.$did not affect $\mathrm{Cx} 43$ expression, but the threshold level of $10 \mathrm{mM} \mathrm{K}^{+}$increased). Taken together with our previous findings, the present demonstrations suggest multiple age-dependent and event-induced (sleep and epileptic seizure) stages of pathomechanisms in ADSHE.

Therefore, the congenital loss-of-function S286L-mutant $\alpha 4 \beta 2$-nAChR generates two functional abnormalities in S286L-TG, GABAergic disinhibition [10,11] and upregulation of the MAPK/Erk signaling pathway. Impaired GABAergic inhibition leads to the relative enhancement of 
glutamatergic transmission in the thalamocortical and hyperdirect pathways in $\alpha 4 \beta 2$-nAChR predominant regions [6-8,12], resulting in the generation of interictal discharge before ADSHE onset [11]. A combination of hyperglutamatergic transmission (repetitive/persistent propagation of discharges) and an upregulated MAPK/Erk signaling pathway contributes to the development of epileptogenesis/ictogenesis via the upregulation of astroglial Cx43.

It is well-known that the majority of ADSHEs (50-60\%) can be controlled by a relatively low dose of carbamazepine, whereas ADSHE with an S284L mutation has a carbamazepine-resistant feature and requires other antiepileptic drugs, such as zonisamide $[4,41,52,53]$. A therapeutically relevant concentration of zonisamide inhibits both the activity and expression of $\mathrm{Cx} 43$ in the astroglial plasma membrane; however, a therapeutically relevant concentration of carbamazepine does not affect these factors $[23,54]$. Therefore, the activated hemichannel in the OFC likely also contributes to the pathophysiology/ictogenesis of carbamazepine-resistant/zonisamide-sensitive ADSHE seizures with S284L-mutations.

\subsection{Neural Circuits Associated with Dystonia Posturing in ADSHE Seizures}

In our previous study, hyperactivated glutamatergic transmission in the thalamic hyperdirect pathway (from motor thalamic nuclei to the STN), but not the glutamatergic transmission abnormalities in the cortical hyperdirect pathway (from the secondary motor cortex to the STN), provided the pathomechanism of nocturnal paroxysmal dystonia [6]. The cortical connectivity to the STN is sparser compared to that to the striatum, but the OFC exhibits higher tract strength for the STN relative to the striatum [17]. A clinical study observed OFC seizures during nocturnal paroxysmal dystonia and episodic nocturnal wandering featuring stereotypical dystonic posturing [13]. In another ADSHE model, S280F-knockin mice strain also exhibited a dystonic/arousal complex, which is a complex between the nocturnal paroxysmal dystonia and nocturnal paroxysmal arousal of ADSHE seizures [55]. These clinical and preclinical findings suggest that hyperactivity in the basal ganglia is possibly involved in ADSHE seizures. Therefore, to explore the pathomechanisms of ADSHE seizures, the present study determined glutamatergic transmission abnormalities in the cortical hyperdirect (OFC-STN) and corticostriatal (OFC-striatum) pathways.

According to our expectations, after ADSHE onset (eight weeks of age), activation of the postsynaptic AMPA/glutamate receptor in the OFC increased L-glutamate release in the STN (cortical hyperdirect pathway) without affecting that in the striatum (corticostriatal pathway) in both wild-type and S286L-TG. Hemichannel activation in the OFC enhanced L-glutamate release in the STN of both wild-type and S286L-TG, but sensitivity to the hemichannel activation of S286L-TG was predominant rather than that of the wild-type. Interestingly, the glutamatergic transmission in the cortical hyperdirect pathway associated with the astroglial hemichannel was observed prior to hemichannel activation in S286L-TG, since CBX inhibited AMPA-evoked L-glutamate release before FCHK-evoked stimulation. Taken together with the upregulation of Cx43 expression in the OFC of S286L-TG, at eight weeks of age, the upregulated Cx43 hemichannel of S286L-TG was probably weakly activated during the interictal stages. Contrary to the hemichannel, in the wild-type, $\alpha 4 \beta 2$-nAChR enhanced AMPA-evoked glutamate release in the cortical hyperdirect pathway under conditions of both hemichannel resting and activation in the OFC, whereas the AMPA-evoked L-glutamate release in the STN of S286L-TG was insensitive to $\alpha 4 \beta 2-n A C h R$ before hemichannel activation. These discrepancies in responses to the $\alpha 4 \beta 2-n A C h R$ of glutamatergic transmission in the hyperdirect pathway between S286L-TG and the wild-type suggest that the loss-of-function S286L-mutant $\alpha 4 \beta 2$-nAChR likely does not directly contribute to the generation of epileptic ADSHE focus in the OFC, but indirectly contributes to the generation of focus via the activation/upregulation of $\mathrm{Cx} 43$ hemichannel hyperactivation.

Similar to the observations at eight weeks of age, the activation of $\alpha 4 \beta 2-n A C h R$ enhanced AMPA-evoked glutamate release in the cortical hyperdirect pathway under conditions of both hemichannel resting and activation in the OFC of the wild-type, whereas before hemichannel activation, the AMPA-evoked L-glutamate release of S286L-TG was insensitive to $\alpha 4 \beta 2-\mathrm{nAChR}$. In contrast to 
$\alpha 4 \beta 2-\mathrm{nAChR}$, the sensitivity levels to the activated hemichannel of glutamatergic transmission in the cortical hyperdirect pathway of S286L-TG showed similar features to the wild-type. Therefore, the differences in the sensitivity of glutamatergic transmission to activated hemichannels before (four weeks of age) and after (eight weeks of age) ADSHE seizure onset suggest that the activation of upregulated Cx43 in the OFC of S286L-TG likely contributes to the development of ictogenesis through the hyperactivation of tripartite synaptic transmission. In other words, the hypersensitivity of hemichannel activity in the OFC to the propagation of discharges (physiological sleep spindle, interictal and ictal discharges) plays important roles in clinical ADSHE features, such as the common occurrence of ADSHE seizures during non-REM sleep phases and once ADSHE seizure leading to subsequent frequent seizures during the same night $[2,13]$.

\section{Materials and Methods}

\subsection{Chemical Agents and Drug Administration}

The (E)-N-Methyl-4-(3-pyridinyl)-3-buten-1-amine oxalate (RJR2403: selective $\alpha 4 \beta 2$-nAChR agonist) was obtained from Cosmo Bio (Tokyo, Japan). The Amino-3-(3-hydroxy-5-methylisoxazol-4-yl)propanoic acid (AMPA: AMPA/glutamate receptor agonist), voltage-dependent sodium channel inhibitor, tetrodotoxin (TTX), and nicotine ditartrate were obtained from Wako Chemicals (Osaka, Japan). Carbenoxolone (CBX: hemichannel inhibitor) and 10-[4'-(N,N-diethylamino)butyl]-2-chlorophenoxazine hydrochloride (10-DEBC: Akt inhibitor) were obtained from Funakoshi (Tokyo, Japan). Moreover, 5-(2-Phenyl-pyrazolo [1,5-a]pyridin-3-yl)-1H-pyrazolo[3,4-c]pyridazin-3-ylamine (FR180204: Erk inhibitor) was obtained from Tokyo Chemical Industry (Tokyo, Japan).

All compounds were prepared on the day of the experiment. In the microdialysis study, AMPA, CBX, TTX, and RJR2403 were dissolved in a modified ringer solution (MRS) composed of the following (in $\mathrm{mM}$ ): $145 \mathrm{Na}^{+}, 2.7 \mathrm{~K}^{+}, 1.2 \mathrm{Ca}^{2+}, 1.0 \mathrm{Mg}^{2+}$, and $154.4 \mathrm{Cl}^{-}$, buffered with $2 \mathrm{mM}$ phosphate buffer and $1.1 \mathrm{mM}$ Tris buffer at $\mathrm{pH} 7.4$ [56-60]. In the primary cultured astrocyte study, FR180204 was initially made as $10 \mathrm{mM}$ stocks in dimethyl sulfoxide and then diluted to Dulbecco's modified Eagle's medium (D6546: Sigma-Aldrich, St. Louis, MO) containing 10\% fetal calf serum (fDMEM). The 10-DEBC was dissolved in fDMEM directly. To study the effects of subchronic nicotine administration on the expression of Cx43, pErk/Erk, and pAkt/Akt in the OFC plasma membrane, rats were subchronically administered with nicotine ditartrate ( $50 \mathrm{mg} / \mathrm{kg} /$ day for 7 days), using a subcutaneous osmotic pump (2ML_1, Alzet, Cupertino, CA; the nominal pumping rate and duration were $10 \mu \mathrm{L} / \mathrm{h}$ over 7 days).

\subsection{Experimental Animals}

Animal care, experimental procedures, and protocols for the animal experiments were approved by the Animal Research Ethics Committee of the Mie University School of Medicine (No. 24-37-R3, 7 March 2018). All studies involving animals were reported in accordance with the ARRIVE guidelines for reporting experiments involving animals. A total 126 rats, wild-type littermates $(n=90)$, and S286L-TG rats $(n=84)[7,61]$ (Sprague Dawley strain background, SLC, Shizuoka, Japan) were maintained in a controlled environment $\left(22 \pm 1{ }^{\circ} \mathrm{C}\right)$ on a $12 \mathrm{~h}$ dark/light cycle and used in the experiments as described. Rats were randomly assigned to the treatment groups of each experiment. All experiments in this study were designed with equally sized animal groups $(n=6)$ without carrying out a formal power analysis, in keeping with previous studies [6-8,12]. Where possible, we sought to randomize and blind the data. In particular, for the determination of L-glutamate and protein levels, the sample order was determined by a random number table.

\subsection{Microdialysis}

Wild-type $(n=60)$ and S286L-TG $(n=60)$ rats (4 and 8 weeks of age) were anesthetized with $1.8 \%$ isoflurane and then placed on a stereotactic frame. Microdialysis studies were prepared, using a 
protocol adapted from previously described methods [6-8,12,54,58,62]. A concentric direct-insertion type dialysis probe ( $0.22 \mathrm{~mm}$ diameter, $3 \mathrm{~mm}$ exposed membrane: Eicom, Kyoto, Japan) was implanted in the $\mathrm{OFC}(\mathrm{A}=+3.2 \mathrm{~mm}, \mathrm{~L}=+2.4 \mathrm{~mm}, \mathrm{~V}=-6.5 \mathrm{~mm}$, relative to bregma) and striatum $(\mathrm{A}=+1.0 \mathrm{~mm}$, $\mathrm{L}=-3.5 \mathrm{~mm}, \mathrm{~V}=-6.0 \mathrm{~mm}$, relative to bregma) [7,62-64]. Another concentric direct-insertion type probe with a shorter exposed membrane $(0.22 \mathrm{~mm}$ diameter, $1 \mathrm{~mm}$ exposed membrane: Eicom) was then implanted in the STN $(A=-3.6 \mathrm{~mm}, \mathrm{~L}=-2.5 \mathrm{~mm}, \mathrm{~V}=-8.4 \mathrm{~mm}$, relative to bregma) $[8,63,65,66]$. Experiments were not started until three consecutive baseline transmitter measurements yielded a coefficient of variation of less than 5\%. Dialysates were then collected for $60 \mathrm{~min}$ (pretreatment period) followed by $180 \mathrm{~min}$ of sampling after AMPA administration [6,12].

For the microdialysis study, to activate the hemichannel function in the OFC, the perfusion medium in the OFC was switched to $\mathrm{Ca}^{2+}$-free with $100 \mathrm{mM} \mathrm{K}^{+}$containing MRS (FCHK-MRS) for $20 \mathrm{~min}$ (FCHK-MRS activation) [6,8] (Figure 1). To explore the effects of the hemichannel and $\alpha 4 \beta 2-n A C h R$ on glutamatergic transmission in the cortical hyperdirect (OFC-STN) and corticostriatal (OFC-striatum) pathways of the wild-type and S286L-TG, the perfusion medium in the OFC began with MRS with or without (control) $100 \mu \mathrm{M}$ CBX (non-selective hemichannel inhibitor), $100 \mu \mathrm{M}$ RJR2406 (selective $\alpha 4 \beta 2$-nAChR agonist), or $100 \mu \mathrm{M}$ CBX plus $100 \mu \mathrm{M}$ RJR2406. The perfusates in the STN and striatum were maintained with MRS alone during the experiment. After stabilization of the L-glutamate level in the STN or striatum, the perfusate in the OFC was switched to MRS containing the same agent with $100 \mu \mathrm{M}$ AMPA for $180 \mathrm{~min}$ (1st AMPA-evoked stimulation). After the 1st AMPA-evoked stimulation, the perfusion medium in the OFC was switched to MRS. After stabilization of the L-glutamate level in the STN or striatum, the perfusion medium in the OFC was switched to FCHK-MRS $\left(\mathrm{Ca}^{2+}{ }_{\text {-free }}\right.$ with $100 \mathrm{mM} \mathrm{K}^{+}$) for $20 \mathrm{~min}$ (hemichannel activation). After stabilization of the L-glutamate level in the STN or striatum, the perfusion medium in the OFC was switched to MRS containing the same agent with $100 \mu \mathrm{M}$ AMPA for 180 min again (2nd AMPA-evoked stimulation) (Figure 1). The time between the 1st and 2nd AMPA-evoked stimulations was around $240 \mathrm{~min}$.

\subsection{Ultra-High-Performance Liquid Chromatography (UHPLC)}

L-glutamate levels were determined by using UHPLC equipped with xLC3185PU (Jasco, Tokyo, Japan) and fluorescence detection (xLC3120FP, Jasco) following dual derivatization with isobutyryl-L-cysteine/o-phthalaldehyde $[8,67,68]$. The derivatized samples (5 $\mu \mathrm{L}$ aliquots) were injected via an autosampler (xLC3059AS, Jasco). The analytical column (YMC Triat C18, particle $1.8 \mu \mathrm{m}$, $50 \times 2.1 \mathrm{~mm}$, YMC, Kyoto, Japan) was maintained at $45^{\circ} \mathrm{C}$, and the flow rate was set to $500 \mu \mathrm{L} / \mathrm{min}$. A linear gradient elution program was used over a period of $10 \mathrm{~min}$ with mobile phases $\mathrm{A}(0.05 \mathrm{M}$ citrate buffer, $\mathrm{pH} 5.0)$ and B (0.05 M citrate buffer containing 30\% acetonitrile and 30\% methanol, $\mathrm{pH}$ 3.5). The excitation/emission wavelengths of the fluorescence detector were set to $280 / 455 \mathrm{~nm}$.

\subsection{Capillary Immunoblotting Analysis}

The capillary immunoblotting analysis was performed, using Wes (ProteinSimple, Santa Clara, CA, USA), according to the ProteinSimple user manual [6-8,12]. The lysates of the primary cultured astrocytes were mixed with a master mix (ProteinSimple) to a final concentration of $1 \times$ sample buffer, $1 \times$ fluorescent molecular weight marker, and $40 \mathrm{mM}$ dithiothreitol and then heated at $95^{\circ} \mathrm{C}$ for $5 \mathrm{~min}$. The samples, blocking reagents, primary antibodies, HRP-conjugated secondary antibodies, chemiluminescent substrate (SuperSignal West Femto: Thermo Fisher Scientific, Waltham, MA, USA), and separation and stacking matrices were also dispensed to the designated wells in a 25 well plate. After plate loading, the separation electrophoresis and immunodetection steps took place in the capillary system and were fully automated. A capillary immunoblotting analysis was carried out at room temperature, and the instrument's default settings were used. Capillaries were first filled with a separation matrix followed by a stacking matrix, with about $40 \mathrm{~nL}$ of the sample used for loading. During electrophoresis, the proteins were separated by molecular weight through the stacking and separation matrices at 250 volts for $40-50 \mathrm{~min}$ and then immobilized on the capillary wall, using proprietary photo-activated capture chemistry. The matrices 
were then washed out. The capillaries were next incubated with a blocking reagent for $15 \mathrm{~min}$, and the target proteins were immunoprobed with primary antibodies followed by HRP-conjugated secondary antibodies (Anti-Rabbit IgG HRP, A00098, $10 \mu \mathrm{g} / \mathrm{mL}$, GenScript, Piscataway, NJ). The antibodies of GAPDH (NB300-322, 1:100, Novus Biologicals, Littleton, CO, USA), Cx43 (C6219, 1:100, Sigma-Aldrich, St. Louis, MO, USA), Erk (AF1576, $10 \mu \mathrm{g} / \mathrm{mL}$, R\&D systems, Minneapolis, MN, USA), pErk (AF1018, $5 \mu \mathrm{g} / \mathrm{mL}$, R\&D systems), Akt (AF1775, $1 \mu \mathrm{g} / \mathrm{mL}$, R\&D systems), and pAkt (AF877, $5 \mu \mathrm{g} / \mathrm{mL}$, R\&D systems) were diluted in an antibody diluent (ProteinSimple).

To study the effects of $\alpha 4 \beta 2$-nAChR on the expression of Cx43 and Erk in the OFC plasma membrane, both wild-type $(n=6)$ and S286L-TG $(n=6)$ at 4 weeks of age (before ADSHE onset) and 12 weeks of age (after ADSHE onset) [7,11] were subchronically administrated nicotine $(50 \mathrm{mg} / \mathrm{kg} / \mathrm{day}$ for 7 days), using a subcutaneous osmotic pump (2ML_1, Alzet) [6,56,57]. The total plasma membrane proteins of the OFC and primary cultured astrocytes were extracted, using a Minute Plasma Membrane Protein Isolation Kit (Invent Biotechnologies, Plymouth, MN) [6].

\subsection{Primary Cultured Astrocytes}

Astrocytes were prepared, using a protocol adapted from previously described methods $[22,54,68]$. Pregnant Sprague-Dawley rats (SLC, Sizuoka, Japan) were housed individually in cages and kept in air-conditioned rooms (temperature, $22 \pm 2{ }^{\circ} \mathrm{C}$ ), with a $12 \mathrm{~h}$ light/dark cycle and free access to food and water. Cultured astrocytes were prepared from the cortical astrocyte cultures of neonatal Sprague-Dawley rats $(n=6)$ sacrificed by decapitation at $0-24 \mathrm{~h}$ of age. Cerebral hemispheres were removed under a dissecting microscope. Tissues were chopped into fine pieces, using scissors and then triturated briefly with a micropipette. The suspension was filtered, using a $70 \mu \mathrm{m}$ nylon mesh (BD, Franklin Lakes, NJ, USA), and centrifuged. Pellets were then re-suspended in fDMEM, which was repeated three times. After culturing for 14 days (DIV14), the contaminating cells were removed via shaking in a standard incubator (BNA-111, Espec, Osaka, Japan) for $16 \mathrm{~h}$ at $200 \mathrm{rpm}$. On DIV21, the astrocytes were removed from the flasks by trypsinization and seeded directly onto a translucent PET membrane $(1.0 \mu \mathrm{m})$ with 24 well plates (BD) at a density of $1 \times 105 \mathrm{cells} / \mathrm{cm}^{2}$ for the experiments. From DIV21 to DIV27, the culture medium (fDMEM) was changed twice a week for 7 days. On DIV27, to study the effects of the extracellular $\mathrm{K}^{+}$level on Cx43 expression in the plasma membrane, the cultured medium was changed (for $6 \mathrm{~h}$ ) to $\mathrm{N}$-fDMEM (control: fDMEM plus $4.6 \mathrm{mM} \mathrm{NaCl}: 5.4 \mathrm{mM} \mathrm{K}^{+}$), MK-fDMEM (fDMEM plus $2.1 \mathrm{mM} \mathrm{KCl}$ and $2.5 \mathrm{mM} \mathrm{NaCl}$ : $7.5 \mathrm{mM} \mathrm{K}^{+}$), and HK-fDMEM (fDMEM plus $4.6 \mathrm{mM} \mathrm{KCl}=10.0 \mathrm{mM} \mathrm{K}^{+}$). The composition of $\mathrm{NaCl}$ and $\mathrm{KCl}$ in fDMEM was modified to maintain isotonicity and ionic strength. To study the effects of Erk and Akt on Cx43 expression in the plasma membrane, the medium was changed (for $6 \mathrm{~h}$ ) to N-fDMEM containing $20 \mu \mathrm{M}$ FR180204 (Erk inhibitor) or $10 \mu \mathrm{M}$ 10-DEBC (Akt inhibitor) for $6 \mathrm{~h}$. On DIV 28, the cultured astrocytes were washed out, using artificial-CSF, and then the total plasma membrane proteins were extracted, using a Minute Plasma Membrane Protein Isolation Kit (Invent Biotechnologies) [6].

\subsection{Data Analysis}

All experiments in this study were designed with equally sized animal groups $(n=6)$, without carrying out a formal power analysis, in keeping with previous studies. All values are expressed as the mean $\pm \mathrm{SD}$, and $p<0.05$ (two-tailed) was considered statistically significant for all tests. Drug levels in acute local and subchronically systemic administrations were selected based on values in previous studies. Where possible, we sought to randomize and blind the data. In particular, for the determination of transmitter levels and protein expression, the sample order on the autosampler and Wes were determined by a random number table.

The regional transmitter concentrations in the STN and striatum were analyzed via a Mauchly's sphericity test followed by a multivariate analysis of variance (MANOVA), using BellCurve for Excel ver. 3.2 (Social Survey Research Information Co., Ltd., Tokyo, Japan). When the data did not violate the assumption of sphericity $(p>0.05)$, the F-value of the MANOVA was analyzed, using 
sphericity-assumed degrees of freedom. However, if the assumption of sphericity was violated $(p<0.05)$, the F-value was analyzed, using Chi-Muller's corrected degrees of freedom. When the F-value for the genotype/drug/time factors of MANOVA was significant, the data were analyzed by a Tukey's multiple comparison test. The transmitter level was expressed as the area under the curve between 20 and 180 min $\left(\mathrm{AUC}_{20-180}\right)$ after the perfusion of AMPA containing MRS. The effects of perfusion with TTX and CBX into the basal L-glutamate release were analyzed, using a one-way analysis of variance (ANOVA) with Tukey's multiple comparison. The protein expression of Cx43, pErk, and pAkt in the plasma membrane fraction was analyzed by a two-way ANOVA with Tukey's multiple comparison, using BellCurve for Excel.

\section{Conclusions}

In conclusion, this study provided evidence for the age-dependent and sleep/seizure-induced multi-stage pathomechanisms of ADSHE with S284L-mutations, using the genetic ADSHE model (S286L-TG). Congenital functional abnormalities and the loss-of-function S286L-mutant $\alpha 4 \beta 2$-nAChR produce GABAergic disinhibition, resulting in enhanced glutamatergic transmission and an upregulated MAPK/Erk signaling pathway (Figure 8). Wild-type $\alpha 4 \beta 2$-nAChR suppresses pErk, but the S286L-mutant $\alpha 4 \beta 2-n A C h R$ impairs the inhibitory function of pErk, resulting in the upregulation of Cx43 expression. Under functional abnormalities, the propagation of physiological (sleep spindle bursts) and pathological (interictal/ictal discharges) discharges to the OFC leads to an event-related enhancement in the function of upregulated $\mathrm{Cx} 43$, resulting in enhanced excitatory tripartite synaptic transmission (Figure 8). Therefore, a combination of secondary functional abnormalities induced by the loss-of-function S286L-mutant $\alpha 4 \beta 2$-nAChR, GABAergic disinhibition, and Cx43 upregulation contributes to the pathomechanism of ADSHE. Interestingly, even if ADSHE seizures are controlled, once a patient experiences an ADSHE seizure, he or she will experience many more ADSHE seizures during that same night. 


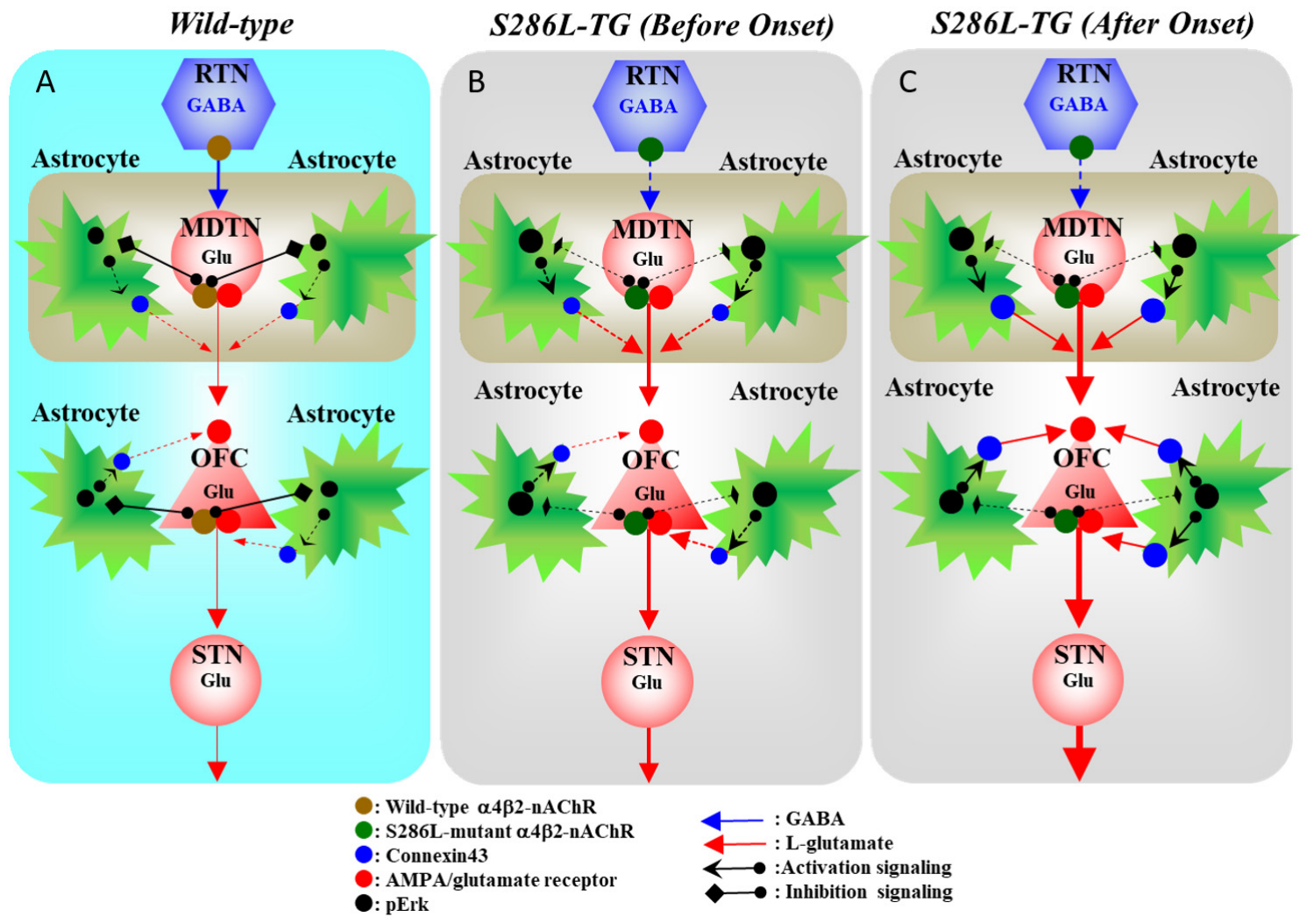

Figure 8. Proposed hypothesis of the multi-stage pathomechanisms of S286L-TG. Proposed hypothesis for the functional abnormalities of glutamatergic transmission in the thalamocortical and cortical hyperdirect pathways in the wild-type (A), S286L-TG before ADSHE onset (B), and after ADSHE onset (C). Reticular thalamic nucleus (RTN) mainly projects GABAergic terminals to various thalamic nuclei, including mediodorsal thalamic nucleus (MDTN). The activation of $\alpha 4 \beta 2$-nAChR in the RTN enhances GABAergic transmission in the RTN-MDTN pathways of the wild-type (A), whereas the S286L-mutant $\alpha 4 \beta 2$-nAChR impairs the activation of GABAergic transmission in the RTN-MDTN in S286L-TG (B,C). MDTN project glutamatergic terminals to the OFC. In the MDTN, both $\alpha 4 \beta 2-\mathrm{nAChR}$ and the AMPA/glutamate receptor activate glutamatergic transmission to the OFC (A-C). Wild-type $\alpha 4 \beta 2-n A C h R$ inhibits astroglial Erk, resulting in the suppression of connexin43 expression in the astroglial plasma membrane (A). Contrary to the wild-type, in S286L-TG, the loss-of-function S286L-mutant $\alpha 4 \beta 2$-nAChR lacks suppressive effects on pErk $(B, C)$ but is insufficient to upregulate connexin43 (B). A combination of the persistent/repetitive propagation of the hyperactivation of glutamatergic transmission in MDTN-OFC induced by the GABAergic disinhibition of S286L-TG and pErk upregulation enhances connexin43 expression.

Author Contributions: Conceptualization, M.O.; data curation, K.F. and M.O.; formal analysis, K.F. and M.O.; funding acquisition, M.O.; methodology, M.O.; project administration, M.O.; validation, K.F.; writing original draft, M.O.; writing review and editing, M.O. All authors have read and agreed to the published version of the manuscript.

Funding: This study was supported by Japan Society for the Promotion of Science (15H04892 and 19K08073).

Conflicts of Interest: The authors state no conflict of interest. 


\section{Abbreviations}

\begin{tabular}{|c|c|}
\hline 10-DEBC & 10-[4'-(N,N-Diethylamino)butyl]-2-chlorophenoxazine hydrochloride \\
\hline Ach & acetylcholine \\
\hline ADSHE & autosomal dominant sleep-related hypermotor epilepsy \\
\hline Akt & protein kinase B \\
\hline AMPA & amino-3-(3-hydroxy-5-methyl-isoxazol-4-yl)propanoic acid \\
\hline ANOVA & analysis of variance \\
\hline AUC & area under curve value \\
\hline $\mathrm{CBX}$ & carbenoxolone \\
\hline CHRNA2 & cholinergic receptor nicotinic alpha 2 subunit \\
\hline CHRNA4 & cholinergic receptor nicotinic alpha 4 subunit \\
\hline CHRNB2 & cholinergic receptor nicotinic beta 2 subunit \\
\hline $\mathrm{CRH}$ & corticotropin releasing hormone \\
\hline Cx43 & connexin43 \\
\hline DEPDC5 & DEP domain containing 5, GATOR1 subcomplex subunit \\
\hline DIV & after culture days \\
\hline EEG & electroencephalogram \\
\hline Erk & extracellular signal-regulated kinase \\
\hline FCHK-MRS & $\mathrm{Ca}^{2+}$-free with $100 \mathrm{mM} \mathrm{K}^{+}$containing modified Ringer's solution \\
\hline fDMEM & Dulbecco's modified Eagle's medium containing $10 \%$ fetal calf serum \\
\hline FR180204 & 5-(2-Phenyl-pyrazolo[1,5-a]pyridin-3-yl)-1H-pyrazolo[3,4-c]pyridazin-3-ylamine \\
\hline HK-fDMEM & Dulbecco's modified Eagle's medium containing $10 \%$ fetal calf serum plus $4.6 \mathrm{mM} \mathrm{KCl}$ \\
\hline KCC & $\mathrm{K}^{+} / 2 \mathrm{Cl}^{-}$cotransporter \\
\hline KCNT1 & potassium sodium-activated channel subfamily T member 1 \\
\hline MANOVA & multivariate analysis of variance \\
\hline MAPK & mitogen-activated protein kinase \\
\hline MDTN & mediodorsal thalamic nucleus \\
\hline MK-fDMEM & $\begin{array}{l}\text { Dulbecco's modified Eagle's medium containing } 10 \% \text { fetal calf serum plus } 2.1 \mathrm{mM} \mathrm{KCl} \text { and } \\
2.5 \mathrm{mM} \mathrm{NaCl}\end{array}$ \\
\hline MRS & modified Ringer's solution \\
\hline mTOR & mammalian target of rapamycin \\
\hline nAChR & nicotinic acetylcholine receptor \\
\hline N-fDMEM & Dulbecco's modified Eagle's medium containing $10 \%$ fetal calf serum plus $4.6 \mathrm{mM} \mathrm{NaCl}$ \\
\hline OFC & orbitofrontal cortex \\
\hline pAkt & phosphorylated protein kinase B \\
\hline pErk & phosphorylated extracellular signal-regulated kinase \\
\hline PI3K & phosphoinositide 3-kinase \\
\hline RJR2403 & (E)-N-Methyl-4-(3-pyridinyl)-3-buten-1-amine oxalate \\
\hline RTN & reticular thalamic nucleus \\
\hline S286L-TG & bearing S286L-mutation in rat Chrna4 gene transgenic rat \\
\hline STN & subthalamic nucleus \\
\hline TSC & tuberous sclerosis complex \\
\hline UHPLC & ultra-high-performance liquid chromatograph \\
\hline
\end{tabular}

\section{References}

1. Scheffer, I.E.; Bhatia, K.P.; Lopes-Cendes, I.; Fish, D.R.; Marsden, C.D.; Andermann, F.; Andermann, E.; Desbiens, R.; Cendes, F.; Manson, J.I.; et al. Autosomal dominant frontal epilepsy misdiagnosed as sleep disorder. Lancet 1994, 343, 515-517. [CrossRef]

2. Tinuper, P.; Bisulli, F.; Cross, J.H.; Hesdorffer, D.; Kahane, P.; Nobili, L.; Provini, F.; Scheffer, I.E.; Tassi, L.; Vignatelli, L.; et al. Definition and diagnostic criteria of sleep-related hypermotor epilepsy. Neurology 2016, 86, 1834-1842. [CrossRef] [PubMed]

3. Hebbar, M.; Mefford, H.C. Recent advances in epilepsy genomics and genetic testing. F1000 Res. 2020, 9, 185. [CrossRef] 
4. Okada, M.; Zhu, G.; Yoshida, S.; Kaneko, S. Validation criteria for genetic animal models of epilepsy. Epilepsy Seizure 2010, 3, 109-120. [CrossRef]

5. Nobili, L.; Proserpio, P.; Combi, R.; Provini, F.; Plazzi, G.; Bisulli, F.; Tassi, L.; Tinuper, P. Nocturnal frontal lobe epilepsy. Curr. Neurol. Neurosci. Rep. 2014, 14, 424. [CrossRef]

6. Fukuyama, K.; Fukuzawa, M.; Ruri, O.; Okada, M. Upregulated Connexin 43 Induced by Loss-of-Functional S284L-Mutant alpha4 Subunit of Nicotinic ACh Receptor Contributes to Pathomechanisms of Autosomal Dominant Sleep-Related Hypermotor Epilepsy. Pharmaceuticals 2020, 13, 58. [CrossRef]

7. Fukuyama, K.; Fukuzawa, M.; Shiroyama, T.; Okada, M. Pathogenesis and pathophysiology of autosomal dominant sleep-related hypermotor epilepsy with S284L-mutant alpha4 subunit of nicotinic ACh receptor. Br. J. Pharmacol. 2020, 177, 2143-2162. [CrossRef]

8. Fukuyama, K.; Fukuzawa, M.; Shiroyama, T.; Okada, M. Pathomechanism of nocturnal paroxysmal dystonia in autosomal dominant sleep-related hypermotor epilepsy with S284L-mutant $\alpha 4$ subunit of nicotinic ACh receptor. Biomed. Pharmacother. 2020, 126, 110070. [CrossRef] [PubMed]

9. Shiba, Y.; Mori, F.; Yamada, J.; Migita, K.; Nikaido, Y.; Wakabayashi, K.; Kaneko, S.; Okada, M.; Hirose, S.; Ueno, S. Spontaneous epileptic seizures in transgenic rats harboring a human ADNFLE missense mutation in the beta2-subunit of the nicotinic acetylcholine receptor. Neurosci. Res. 2015, 100, 46-54. [CrossRef]

10. Yamada, J.; Zhu, G.; Okada, M.; Hirose, S.; Yoshida, S.; Shiba, Y.; Migita, K.; Mori, F.; Sugawara, T.; Chen, L.; et al. A novel prophylactic effect of furosemide treatment on autosomal dominant nocturnal frontal lobe epilepsy (ADNFLE). Epilepsy Res. 2013, 107, 127-137. [CrossRef]

11. Zhu, G.; Okada, M.; Yoshida, S.; Ueno, S.; Mori, F.; Takahara, T.; Saito, R.; Miura, Y.; Kishi, A.; Tomiyama, M.; et al. Rats harboring S284L Chrna4 mutation show attenuation of synaptic and extrasynaptic GABAergic transmission and exhibit the nocturnal frontal lobe epilepsy phenotype. J. Neurosci. 2008, 28, 12465-12476. [CrossRef]

12. Fukuyama, K.; Fukuzawa, M.; Okada, M. Upregulated and hyperactivated thalamic connexin 43 plays important roles in pathomechanisms of cognitive impairment and seizure of autosomal dominant sleep-related hypermotor epilepsy with S284L-mutant $\alpha 4$ subunit of nicotinic ACh receptor. Pharmaceuticals 2020, 13, 99. [CrossRef] [PubMed]

13. Provini, F.; Plazzi, G.; Tinuper, P.; Vandi, S.; Lugaresi, E.; Montagna, P. Nocturnal frontal lobe epilepsy. A clinical and polygraphic overview of 100 consecutive cases. Brain A J. Neurol. 1999, 122, 1017-1031. [CrossRef]

14. Picard, F.; Bruel, D.; Servent, D.; Saba, W.; Fruchart-Gaillard, C.; Schollhorn-Peyronneau, M.A.; Roumenov, D.; Brodtkorb, E.; Zuberi, S.; Gambardella, A.; et al. Alteration of the in vivo nicotinic receptor density in ADNFLE patients: A PET study. Brain A J. Neurol. 2006, 129, 2047-2060. [CrossRef]

15. Cavdar, S.; Ozgur, M.; Cakmak, Y.O.; Kuvvet, Y.; Kunt, S.K.; Saglam, G. Afferent projections of the subthalamic nucleus in the rat: Emphasis on bilateral and interhemispheric connections. Acta Neurobiol. Exp. 2018, 78, 251-263.

16. Voorn, P.; Vanderschuren, L.J.; Groenewegen, H.J.; Robbins, T.W.; Pennartz, C.M. Putting a spin on the dorsal-ventral divide of the striatum. Trends Neurosci. 2004, 27, 468-474. [CrossRef] [PubMed]

17. Isaacs, B.R.; Forstmann, B.U.; Temel, Y.; Keuken, M.C. The Connectivity Fingerprint of the Human Frontal Cortex, Subthalamic Nucleus, and Striatum. Front. Neuroanat. 2018, 12, 60. [CrossRef]

18. Okada, M.; Fukuyama, K.; Shiroyama, T.; Murata, M. A Working Hypothesis Regarding Identical Pathomechanisms between Clinical Efficacy and Adverse Reaction of Clozapine via the Activation of Connexin43. J. Mol. Neurosci. 2020, 21, 7019. [CrossRef]

19. Medina-Ceja, L.; Salazar-Sanchez, J.C.; Ortega-Ibarra, J.; Morales-Villagran, A. Connexins-Based Hemichannels/Channels and Their Relationship with Inflammation, Seizures and Epilepsy. Int. J. Mol. Sci. 2019, 20, 5976. [CrossRef]

20. Kar, R.; Batra, N.; Riquelme, M.A.; Jiang, J.X. Biological role of connexin intercellular channels and hemichannels. Arch. Biochem. Biophys. 2012, 524, 2-15. [CrossRef]

21. Fasciani, I.; Temperan, A.; Perez-Atencio, L.F.; Escudero, A.; Martinez-Montero, P.; Molano, J.; Gomez-Hernandez, J.M.; Paino, C.L.; Gonzalez-Nieto, D.; Barrio, L.C. Regulation of connexin hemichannel activity by membrane potential and the extracellular calcium in health and disease. Neuropharmacology 2013, 75, 479-490. [CrossRef] [PubMed] 
22. Fukuyama, K.; Okada, M. Effects of levetiracetam on astroglial release of kynurenine-pathway metabolites. Br. J. Pharmacol. 2018, 175, 4253-4265. [CrossRef]

23. Fukuyama, K.; Ueda, Y.; Okada, M. Effects of Carbamazepine, Lacosamide and Zonisamide on Gliotransmitter Release Associated with Activated Astroglial Hemichannels. Pharmaceuticals 2020, 13, 117. [CrossRef] [PubMed]

24. Schuller, H.M. Is cancer triggered by altered signalling of nicotinic acetylcholine receptors. Nat. Rev. Cancer 2009, 9, 195-205. [CrossRef]

25. Akaike, A.; Izumi, Y. Overview. In Nicotinic Acetylcholine Receptor Signaling in Neuroprotection; Akaike, A., Shimohama, S., Misu, Y., Eds.; Springer: Singapore, 2018; pp. 1-16. [CrossRef]

26. Li, S.; Guan, S.; Wang, Y.; Cheng, L.; Yang, Q.; Tian, Z.; Zhao, M.; Wang, X.; Feng, B. Nicotine inhibits rapamycin-induced pain through activating mTORC1/S6K/IRS-1-related feedback inhibition loop. Brain Res. Bull. 2019, 149, 75-85. [CrossRef]

27. Yang, J.; Long, Y.; Xu, D.M.; Zhu, B.L.; Deng, X.J.; Yan, Z.; Sun, F.; Chen, G.J. Age- and Nicotine-Associated Gene Expression Changes in the Hippocampus of APP/PS1 Mice. J. Mol. Neurosci. 2019, 69, 608-622. [CrossRef]

28. Ribeiro-Rodrigues, T.M.; Martins-Marques, T.; Morel, S.; Kwak, B.R.; Girao, H. Role of connexin 43 in different forms of intercellular communication gap junctions, extracellular vesicles and tunnelling nanotubes. J. Cell. Sci. 2017, 130, 3619-3630. [CrossRef] [PubMed]

29. Panet, R.; Eliash, M.; Atlan, H. Na+/K+/Cl- cotransporter activates MAP-kinase cascade downstream to protein kinase C, and upstream to MEK. J. Cell. Physiol. 2006, 206, 578-585. [CrossRef]

30. Warn-Cramer, B.J.; Lampe, P.D.; Kurata, W.E.; Kanemitsu, M.Y.; Loo, L.W.; Eckhart, W.; Lau, A.F. Characterization of the mitogen-activated protein kinase phosphorylation sites on the connexin-43 gap junction protein. J. Biol. Chem. 1996, 271, 3779-3786. [CrossRef]

31. Lampe, P.D.; TenBroek, E.M.; Burt, J.M.; Kurata, W.E.; Johnson, R.G.; Lau, A.F. Phosphorylation of connexin 43 on serine368 by protein kinase $C$ regulates gap junctional communication. J. Cell. Biol. 2000, 149, 1503-1512. [CrossRef]

32. Chen, W.; Feng, J.; Tong, W. Phosphorylation of astrocytic connexin 43 by ERK1/2 impairs blood-brain barrier in acute cerebral ischemia. Cell. Biosci. 2017, 7, 43. [CrossRef] [PubMed]

33. Ock, S.; Lee, W.S.; Kim, H.M.; Park, K.S.; Kim, Y.K.; Kook, H.; Park, W.J.; Lee, T.J.; Abel, E.D.; Kim, J. Connexin43 and zonula occludens-1 are targets of Akt in cardiomyocytes that correlate with cardiac contractile dysfunction in Akt deficient hearts. Biochim. Biophys. Acta Mol. Basis Dis. 2018, 1864, 1183-1191. [CrossRef] [PubMed]

34. Zhao, M.; Hou, S.; Feng, L.; Shen, P.; Nan, D.; Zhang, Y.; Wang, F.; Ma, D.; Feng, J. Vinpocetine Protects Against Cerebral Ischemia-Reperfusion Injury by Targeting Astrocytic Connexin43 via the PI3K/AKT Signaling Pathway. Front. Neurosci. 2020, 14, 223. [CrossRef]

35. Kume, T.; Takada-Takatori, Y. Nicotinic Acetylcholine Receptor Signaling: Roles in Neuroprotection. In Nicotinic Acetylcholine Receptor Signaling in Neuroprotection; Akaike, A., Shimohama, S., Misu, Y., Eds.; Springer: Singapore, 2018; pp. 59-71. [CrossRef]

36. Larsen, H.M.; Hansen, S.K.; Mikkelsen, J.D.; Hyttel, P.; Stummann, T.C. Alpha7 nicotinic acetylcholine receptors and neural network synaptic transmission in human induced pluripotent stem cell-derived neurons. Stem. Cell. Res. 2019, 41, 101642. [CrossRef] [PubMed]

37. Fukuyama, K.; Okubo, R.; Murata, M.; Shiroyama, T.; Okada, M. Activation of Astroglial Connexin is Involved in Concentration-Dependent Double-Edged Sword Clinical Action of Clozapine. Cells 2020, 9, 414. [CrossRef]

38. Blomstrand, F.; Venance, L.; Siren, A.L.; Ezan, P.; Hanse, E.; Glowinski, J.; Ehrenreich, H.; Giaume, C. Endothelins regulate astrocyte gap junctions in rat hippocampal slices. Eur. J. Neurosci. 2004, 19, 1005-1015. [CrossRef]

39. Tovar, K.R.; Maher, B.J.; Westbrook, G.L. Direct actions of carbenoxolone on synaptic transmission and neuronal membrane properties. J. Neurophysiol. 2009, 102, 974-978. [CrossRef]

40. Yamamura, S.; Ohoyama, K.; Hamaguchi, T.; Nakagawa, M.; Suzuki, D.; Matsumoto, T.; Motomura, E.; Tanii, H.; Shiroyama, T.; Okada, M. Effects of zotepine on extracellular levels of monoamine, GABA and glutamate in rat prefrontal cortex. Br. J. Pharmacol. 2009, 157, 656-665. [CrossRef] 
41. Okada, M.; Yoshida, S.; Zhu, G.; Hirose, S.; Kaneko, S. Biphasic actions of topiramate on monoamine exocytosis associated with both soluble $\mathrm{N}$-ethylmaleimide-sensitive factor attachment protein receptors and $\mathrm{Ca}(2+)$-induced $\mathrm{Ca}(2+)$-releasing systems. Neuroscience 2005, 134, 233-246. [CrossRef]

42. Tanahashi, S.; Yamamura, S.; Nakagawa, M.; Motomura, E.; Okada, M. Dopamine D2 and serotonin 5-HT1A receptors mediate the actions of aripiprazole in mesocortical and mesoaccumbens transmission. Neuropharmacology 2012, 62, 765-774. [CrossRef]

43. Okada, M.; Zhu, G.; Yoshida, S.; Hirose, S.; Kaneko, S. Protein kinase associated with gating and closing transmission mechanisms in temporoammonic pathway. Neuropharmacology 2004, 47, 485. [CrossRef]

44. Flores, C.E.; Nannapaneni, S.; Davidson, K.G.; Yasumura, T.; Bennett, M.V.; Rash, J.E.; Pereda, A.E. Trafficking of gap junction channels at a vertebrate electrical synapse in vivo. Proc. Natl. Acad. Sci. USA 2012, 109, E573CE582. [CrossRef]

45. Duerrschmidt, N.; Hagen, A.; Gaertner, C.; Wermke, A.; Nowicki, M.; Spanel-Borowski, K.; Stepan, H.; Mohr, F.W.; Dhein, S. Nicotine effects on human endothelial intercellular communication via alpha4beta2 and alpha3beta2 nicotinic acetylcholine receptor subtypes. Naunyn Schmiedebergs Arch. Pharmacol. 2012, 385, 621-632. [CrossRef] [PubMed]

46. Cushing, P.; Bhalla, R.; Johnson, A.M.; Rushlow, W.J.; Meakin, S.O.; Belliveau, D.J. Nerve growth factor increases connexin 43 phosphorylation and gap junctional intercellular communication. J. Neurosci. Res. 2005, 82, 788-801. [CrossRef] [PubMed]

47. Hodges, S.L.; Lugo, J.N. Therapeutic role of targeting mTOR signaling and neuroinflammation in epilepsy. Epilepsy Res. 2020, 161, 106282. [CrossRef]

48. Talos, D.M.; Jacobs, L.M.; Gourmaud, S.; Coto, C.A.; Sun, H.; Lim, K.C.; Lucas, T.H.; Davis, K.A.; Martinez-Lage, M.; Jensen, F.E. Mechanistic target of rapamycin complex 1 and 2 in human temporal lobe epilepsy. Ann. Neurol. 2018, 83, 311-327. [CrossRef]

49. Rodrigues-Pinguet, N.; Jia, L.; Li, M.; Figl, A.; Klaassen, A.; Truong, A.; Lester, H.A.; Cohen, B.N. Five ADNFLE mutations reduce the $\mathrm{Ca} 2+$ dependence of the mammalian alpha4beta2 acetylcholine response. J. Physiol. 2003, 550, 11-26. [CrossRef]

50. Kawata, Y.; Okada, M.; Murakami, T.; Mizuno, K.; Wada, K.; Kondo, T.; Kaneko, S. Effects of zonisamide on $\mathrm{K}+$ and $\mathrm{Ca} 2+$ evoked release of monoamine as well as $\mathrm{K}+$ evoked intracellular Ca2+ mobilization in rat hippocampus. Epilepsy Res. 1999, 35, 173-182. [CrossRef]

51. Carmignoto, G.; Haydon, P.G. Astrocyte calcium signaling and epilepsy. Glia 2012, 60, 1227-1233. [CrossRef]

52. Asioli, G.M.; Rossi, S.; Bisulli, F.; Licchetta, L.; Tinuper, P.; Provini, F. Therapy in Sleep-Related Hypermotor Epilepsy (SHE). Curr. Treat. Options Neurol. 2020, 22, 1. [CrossRef] [PubMed]

53. Miyajima, T.; Kumada, T.; Saito, K.; Fujii, T. Autism in siblings with autosomal dominant nocturnal frontal lobe epilepsy. Brain Dev. 2013, 35, 155-157. [CrossRef]

54. Okada, M.; Fukuyama, K.; Shiroyama, T.; Ueda, Y. Carbamazepine Attenuates Astroglial L-Glutamate Release Induced by Pro-Inflammatory Cytokines via Chronically Activation of Adenosine A2A Receptor. Int. J. Mol. Sci. 2019, 20, 3727. [CrossRef] [PubMed]

55. Teper, Y.; Whyte, D.; Cahir, E.; Lester, H.A.; Grady, S.R.; Marks, M.J.; Cohen, B.N.; Fonck, C.; McClure-Begley, T.; McIntosh, J.M.; et al. Nicotine-induced dystonic arousal complex in a mouse line harboring a human autosomal-dominant nocturnal frontal lobe epilepsy mutation. J. Neurosci. 2007, 27, 10128-10142. [CrossRef]

56. Okada, M.; Fukuyama, K.; Shiroyama, T.; Ueda, Y. Lurasidone inhibits NMDA antagonist-induced functional abnormality of thalamocortical glutamatergic transmission via 5-HT7 receptor blockade. Br. J. Pharmacol. 2019, 176, 4002-4018. [CrossRef]

57. Okada, M.; Fukuyama, K.; Okubo, R.; Shiroyama, T.; Ueda, Y. Lurasidone Sub-Chronically Activates Serotonergic Transmission via Desensitization of 5-HT1A and 5-HT7 Receptors in Dorsal Raphe Nucleus. Pharmaceuticals 2019, 12, 149. [CrossRef] [PubMed]

58. Okada, M.; Okubo, R.; Fukuyama, K. Vortioxetine Subchronically Activates Serotonergic Transmission via Desensitization of Serotonin 5-HT1A Receptor with 5-HT3 Receptor Inhibition in Rats. Int. J. Mol. Sci. 2019, 20, 6235. [CrossRef] [PubMed]

59. Nakano, T.; Hasegawa, T.; Suzuki, D.; Motomura, E.; Okada, M. Amantadine Combines Astroglial System Xc Activation with Glutamate/NMDA Receptor Inhibition. Biomolecules 2019, 9, 191. [CrossRef] [PubMed] 
60. Okada, M.; Kawata, Y.; Murakami, T.; Wada, K.; Mizuno, K.; Kaneko, S. Interaction between purinoceptor subtypes on hippocampal serotonergic transmission using in vivo microdialysis. Neuropharmacology 1999, 38, 707-715. [CrossRef]

61. Okada, M. Mammalian Expression Plasmids for Production of Transgenic Rat Model of Autosomal Dominant Sleep-related Hypermotor Epilepsy. Available online: https://www.addgene.org/plasmids/articles/28207545/ (accessed on 1 May 2020).

62. Okada, M.; Fukuyama, K.; Kawano, Y.; Shiroyama, T.; Suzuki, D.; Ueda, Y. Effects of acute and sub-chronic administrations of guanfacine on catecholaminergic transmissions in the orbitofrontal cortex. Neuropharmacology 2019, 156, 107547. [CrossRef]

63. Paxinos, G.; Watson, C. The Rat Brain: In Stereotoxic Coordinates, 6th ed.; Academic Press: San Diego, CA, USA, 2007.

64. Yamamura, S.; Ohoyama, K.; Nagase, H.; Okada, M. Zonisamide enhances delta receptor-associated neurotransmitter release in striato-pallidal pathway. Neuropharmacology 2009, 57, 322-331. [CrossRef]

65. Fukuyama, K.; Tanahashi, S.; Hoshikawa, M.; Shinagawa, R.; Okada, M. Zonisamide regulates basal ganglia transmission via astroglial kynurenine pathway. Neuropharmacology 2014, 76 Pt A, 137-145. [CrossRef] [PubMed]

66. Yamamura, S.; Saito, H.; Suzuki, N.; Kashimoto, S.; Hamaguchi, T.; Ohoyama, K.; Suzuki, D.; Kanehara, S.; Nakagawa, M.; Shiroyama, T.; et al. Effects of zonisamide on neurotransmitter release associated with inositol triphosphate receptors. Neurosci. Lett. 2009, 454, 91-96. [CrossRef]

67. Tanahashi, S.; Ueda, Y.; Nakajima, A.; Yamamura, S.; Nagase, H.; Okada, M. Novel delta1-receptor agonist KNT-127 increases the release of dopamine and L-glutamate in the striatum, nucleus accumbens and median pre-frontal cortex. Neuropharmacology 2012, 62, 2057-2067. [CrossRef]

68. Yamamura, S.; Hoshikawa, M.; Dai, K.; Saito, H.; Suzuki, N.; Niwa, O.; Okada, M. ONO-2506 inhibits spike-wave discharges in a genetic animal model without affecting traditional convulsive tests via gliotransmission regulation. Br. J. Pharmacol. 2013, 168, 1088-1100. [CrossRef] [PubMed]

Publisher's Note: MDPI stays neutral with regard to jurisdictional claims in published maps and institutional affiliations.

(C) 2020 by the authors. Licensee MDPI, Basel, Switzerland. This article is an open access article distributed under the terms and conditions of the Creative Commons Attribution (CC BY) license (http://creativecommons.org/licenses/by/4.0/). 